\title{
The subgrid-scale scalar variance under supercritical pressure conditions
}

\author{
Enrica Masi ${ }^{1}$ and Josette Bellan ${ }^{1,2, a)}$ \\ ${ }^{1}$ California Institute of Technology, Pasadena, California 91125, USA \\ ${ }^{2}$ Jet Propulsion Laboratory, California Institute of Technology, Pasadena, California 91109-8099, USA
}

(Received 23 February 2011; accepted 9 June 2011; published online 1 August 2011)

\begin{abstract}
To model the subgrid-scale (SGS) scalar variance under supercritical-pressure conditions, an equation is first derived for it. This equation is considerably more complex than its equivalent for atmospheric-pressure conditions. Using a previously created direct numerical simulation (DNS) database of transitional states obtained for binary-species systems in the context of temporal mixing layers, the activity of terms in this equation is evaluated, and it is found that some of these new terms have magnitude comparable to that of governing terms in the classical equation. Most prominent among these new terms are those expressing the variation of diffusivity with thermodynamic variables and Soret terms having dissipative effects. Since models are not available for these new terms that would enable solving the SGS scalar variance equation, the adopted strategy is to directly model the SGS scalar variance. Two models are investigated for this quantity, both developed in the context of compressible flows. The first one is based on an approximate deconvolution approach and the second one is a gradient-like model which relies on a dynamic procedure using the Leonard term expansion. Both models are successful in reproducing the SGS scalar variance extracted from the filtered DNS database, and moreover, when used in the framework of a probability density function (PDF) approach in conjunction with the $\beta$-PDF, they excellently reproduce a filtered quantity which is a function of the scalar. For the dynamic model, the proportionality coefficient spans a small range of values through the layer cross-stream coordinate, boding well for the stability of large eddy simulations using this model.

(C) 2011 American Institute of Physics. [doi:10.1063/1.3609282]
\end{abstract}

\section{INTRODUCTION}

One of the most useful concepts in turbulent combustion is that of a "flamelet." Its usefulness stems from the fact that the flamelet model is applicable in conjunction with finite rate chemistry, and it is the only turbulent combustion model which contains the essential coupling between classically computed molecular mass-diffusion and chemical reaction rate. This model is applicable when the chemical reactions have a relatively short characteristic time with respect to the flow, so that the flame is thin and turbulent eddies move it and distort it as an entity rather then penetrating into the flame and affecting there the reaction. When the flame is not penetrated by turbulence, the interior of the flame is akin to a laminar flame, and turbulence modeling is only needed for the flow external to the flame; this is a substantial advantage for modeling of turbulent reactive flows. Finding the evolution of the flame is then reduced to obtaining its statistical position. For non-premixed combustion, several assumptions and mathematical manipulations of the governing equations lead to an equation devoid of chemical sources for a quantity which is thus a conserved scalar. This quantity is called "the mixture fraction," $Z$, and the statistics of the stoichiometric value of $Z, Z_{s}$, determines the flame location; whereas, the temporal solution of the species mass fraction, $Y$, and temperature, $T$, in the $Z$ coordinate system

\footnotetext{
a) Telephone: 1-818-354-6959. FAX: 1-818-393-6682. Electronic mail:
} josette.bellan@jpl.nasa.gov. determines the internal flame structure. In the species and energy equations written in the $Z$ coordinate system, the scalar dissipation, $\chi$, acts as a diffusion coefficient. This scalar dissipation is that within the flamelet and thus is at a scale smaller than the solution of the flow field; it is thus the subgrid-scale (SGS) scalar dissipation. Generally, it is hypothesized that $Z$ assumes the form of a $\beta$ probability density function $(\mathrm{PDF}){ }^{2,3}$ and thus finding this distribution reduces to obtaining its mean and variance. Summarizing, in order to utilize the flamelet model, one must have available the mean and variance of $Z$ and the SGS $\chi$. In the context of large eddy simulation (LES) in which the filtered governing equations are solved subject to models included to introduce palliatives for the filtered-out SGS effects, the mean $Z$ value is known from the LES solution. However, neither the SGS $Z$ variance nor the SGS dissipation of $Z$ can be found from the LES solution, and thus they must be modeled. For example, several investigators ${ }^{4,5}$ modeled the SGS scalar variance as proportional to the resolved (i.e., LES) scalar dissipation, a model which follows from the classical mixing length assumption considering that the scalar variance is a measure of the level of scalar fluctuations.

Given the aforementioned importance of the SGS scalar variance in modeling combustion and the numerous studies devoted to solving the SGS scalar variance equation by modeling those of its terms that are not computable from the LES solution (e.g., Refs. 6 and 7), we are here investigating the modeling of the SGS scalar variance under supercritical pressure conditions and use a previously created direct 
numerical simulations (DNS) database for examining the capability of several models. The difference between this situation and the much studied atmospheric-pressure conditions is that here the species mass fluxes include Soret effects, that real gas equations of state (EOSs) instead of the perfect gas EOS are used and that the transport properties are all complex functions of the thermodynamic quantities. In Sec. II we derive the SGS scalar variance equation and show that it includes a large number of subgrid terms the modeling of which is uncertain. Considering this uncertainty, we next examine in Sec. III the conserved scalar extracted from the DNS database and explore whether it can be represented in LES by a presumed PDF for which the SGS scalar variance would be needed. Further, in Sec. IV, we assess the ability of two models to accurately portray the SGS scalar variance. The databases are summarized in Sec. V. Model assessments are here performed on these databases representing transitional states obtained from simulating mixing of two chemical species under supercritical pressure. The database consists of three sets of species and was created in the context of a temporal mixing layer. The mixing situation has been here selected rather than a chemically reacting case because the former represents a conservative choice in that the scalar could have a considerably more complex distribution in a reactive flow due to coupling among thermodynamic variables that would introduce increased flow structure, so that if a model is not accurate for the mixing situation, it will certainly be even less accurate for a reactive flow. On the other hand, an acceptable model describing the conserved scalar only during mixing may not be acceptable for reactive flows, but it represents the departing point for constructing such a model. Additionally, one commonality among the evolution of the flow for all layers was the formation of high density-gradient magnitude (HDGM) regions populating the entire flow, which originate from the combination of the distortion of the original boundary separating the two fluids and the mixing of species having disparate molar masses. These HDGM regions are the sites where mixing between species occurs. Thus, the conserved scalar, which is any of the two species in a layer, displays high nonuniformities and the HDGM regions resemble in geometry the flamelets much studied in reactive flows; this resemblance adds relevance to the databases utilized in this study. Results are presented in Sec. VI. A summary and conclusions are offered in Sec. VII.

\section{THE SGS SCALAR VARIANCE TRANSPORT EQUATION}

Considering $Z$ to be one of the species, the SGS scalar variance is

$$
\sigma_{Z}=\left\{Z^{2}\right\}-\{Z\}^{2},
$$

where the operator $\{*\}$ denotes Favre-filtered quantities (we depart in this section from the typical $\widetilde{Z}$ notation given the complex expressions in the SGS scalar variance equation below), i.e.,

$$
\{Z\}=\frac{\overline{\rho Z}}{\bar{\rho}},
$$

where $\rho$ is the mixture density, and for any function $g(\mathbf{x})$ the filtered value is expressed in physical space by

$$
\bar{g}(\mathbf{x})=\int_{\Omega} g\left(\mathbf{x}^{\prime}\right) \bar{G}\left(\mathbf{x}-\mathbf{x}^{\prime}\right) d \mathbf{x}^{\prime},
$$

where $\bar{G}$ is the filter function associated with the characteristic filter size $\bar{\Delta}$ and $\Omega$ is the entire spatial domain. According to the scalar equation under supercritical conditions, ${ }^{8-12}$ the transport equation of the SGS scalar variance is

$$
\begin{aligned}
& \bar{\rho} \frac{D \sigma_{Z}}{D t}=\underbrace{\nabla \cdot\left[\bar{\rho}\left\{D \alpha_{D}\right\} \nabla \sigma_{Z}\right]}_{\text {term } 1} \underbrace{+2 \bar{\rho}\left\{D \alpha_{D}\right\} \nabla\{Z\} \cdot \nabla\{Z\}}_{\text {term } 2} \underbrace{-2 \bar{\rho}\left[\left\{D \alpha_{D}\right\}\{\nabla Z \cdot \nabla Z\}\right]}_{\text {term } 3} \underbrace{-\nabla \cdot(\bar{\rho} \boldsymbol{\omega}}_{\text {term } 4}) \underbrace{+2\{Z\} \nabla \cdot(\bar{\rho} \boldsymbol{\eta})}_{\text {term } 5} \\
& \begin{array}{l}
\underbrace{-2 \bar{\rho}\left[\left\{D \alpha_{D} \nabla Z \cdot \nabla Z\right\}-\left\{D \alpha_{D}\right\}\{\nabla Z \cdot \nabla Z\}\right]}_{\text {term } 6} \underbrace{+2 \nabla \cdot\left[\bar{\rho}\left\{D \alpha_{D} Z \nabla Z\right\}-\bar{\rho}\left\{D \alpha_{D}\right\}\{Z\right.}_{\text {term } 7} \\
\underbrace{-2\{Z\} \nabla \cdot\left[\bar{\rho}\left\{D \alpha_{D} \nabla Z\right\}-\bar{\rho}\left\{D \alpha_{D}\right\} \nabla\{Z\}\right]} \underbrace{+2 \nabla \cdot\left[\bar{\rho}\left\{\alpha_{B K}(1-Z) Z^{2} \frac{D}{T} \nabla T\right\}\right]}
\end{array} \\
& \text { term } 8 \\
& \text { term } 9 \\
& \underbrace{-2 \bar{\rho}\left\{\alpha_{B K}(1-Z) Z \frac{D}{T} \nabla T \cdot \nabla Z\right\}} \underbrace{+2 \nabla \cdot\left[\bar{\rho}\left\{D \frac{(1-Z) Z^{2}}{R_{u} T} \frac{m_{1} m_{2}}{m} \Lambda \nabla p\right\}\right]} \underbrace{-2 \bar{\rho}\left\{D \frac{(1-Z) Z}{R_{u} T} \frac{m_{1} m_{2}}{m} \Lambda \nabla p \cdot \nabla Z\right\}} \\
& \text { term } 10 \quad \text { term } 11 \quad \text { term } 12 \\
& \underbrace{-2\{Z\} \nabla \cdot\left[\bar{\rho}\left\{\alpha_{B K}(1-Z) Z \frac{D}{T} \nabla T\right\}\right]} \underbrace{-2\{Z\} \nabla \cdot\left[\bar{\rho}\left\{D \frac{(1-Z) Z}{R_{u} T} \frac{m_{1} m_{2}}{m} \Lambda \nabla p\right\}\right]}, \\
& \text { term } 13 \\
& \text { term } 14
\end{aligned}
$$


where $\frac{D \sigma_{Z}}{D t}=\frac{\partial \sigma_{Z}}{\partial t}+\{\mathbf{u}\} \cdot \nabla \sigma_{Z}$ is the material derivative, $t$ is time, $\mathbf{u}$ denotes the velocity, $p$ is the pressure, $T$ is the temperature, $R_{u}$ is the universal gas constant, $D$ and $\alpha_{D}$ are the variable diffusion coefficient and mass diffusion factor, respectively, $\alpha_{B K}$ is the Bearman-Kirkwood form of the thermal diffusion factor, and $\Lambda$ is defined as

$$
\Lambda=\left(\frac{1}{m_{2}} \frac{\partial(m / \rho)}{\partial X_{2}}-\frac{1}{m_{1}} \frac{\partial(m / \rho)}{\partial X_{1}}\right)
$$

with $m$ denoting the molar mass and $X$ labeling the molar fraction (for both $m$ and $X$, subscript 2 refers to $Z$ and subscript 1 refers to the complement of $Z$ in the mixture, $(1-Z)$ ). Vectors $\boldsymbol{\omega}$ and $\boldsymbol{\eta}$ are the SGS fluxes

$$
\begin{gathered}
\omega=\left\{Z^{2} \mathbf{u}\right\}-\left\{Z^{2}\right\}\{\mathbf{u}\}, \\
\boldsymbol{\eta}=\{Z \mathbf{u}\}-\{Z\}\{\mathbf{u}\} .
\end{gathered}
$$

Equation (4) clearly differs from the classical SGS-scalarvariance transport equation under atmospheric conditions (e.g., Jiménez et $a l^{6}{ }^{6}$ ). On the right hand side (r.h.s.) of Eq. (4), one recognizes familiar terms such as the molecular diffusion (term 1), the Fick-issued resolved part of the filtered scalar dissipation and filtered scalar dissipation rate (terms 2 and 3, respectively), and the transport of the square of the scalar and of the scalar by the SGS turbulence (terms 4 and 5, respectively); however, new terms appear as a consequence of the spatial variation of $D \alpha_{D}$ under supercritical conditions and also because of filtering the nonlinear pressure-gradient and Soret terms. We distinguish here between the total dissipation, which is the irreversible entropy production $^{13}$

$$
\chi \propto \mathbf{J}_{\alpha} \cdot \mathbf{J}_{\alpha}
$$

and which is the sum of six terms (since the species massdiffusion flux, $\mathbf{J}_{\alpha}$, is the sum of three terms ${ }^{8,9,13}$ ), and the Fick-issued dissipation which represents only one of these six terms.

Whereas the form of Eq. (4) highlights the new terms due to the supercritical-pressure aspect, another form of the SGS scalar variance equation can highlight new SGS contributions to the SGS scalar variance; this latter form is similar to that of Pera et al. ${ }^{7}$

$$
\begin{aligned}
& \bar{\rho} \frac{D \sigma_{Z}}{D t}=\underbrace{\nabla \cdot\left[\bar{\rho}\left\{D \alpha_{D}\right\} \nabla \sigma_{Z}\right]}_{\text {Resolved Molecular Diffusion }} \underbrace{-\nabla \cdot[\bar{\rho}(\boldsymbol{\omega}-2\{Z\} \boldsymbol{\eta})]}_{\text {SGS Turbulent Fluxes }} \underbrace{-2 \bar{\rho}\left\{D \alpha_{D}\right\}[\{\nabla Z \cdot \nabla Z\}-\nabla\{Z\} \cdot \nabla\{Z\}]}_{\text {Fick-issued SGS Dissipation }} \underbrace{-2 \bar{\rho} \boldsymbol{\eta} \cdot \nabla\{Z\}}_{\text {SGS Production }} \\
& \underbrace{+2 \nabla \cdot\left[\bar{\rho}\left\{D \alpha_{D} Z \nabla Z\right\}-\bar{\rho}\left\{D \alpha_{D}\right\}\{Z \nabla Z\}\right]} \underbrace{-2 \nabla \cdot\left[\bar{\rho}\{Z\}\left(\left\{D \alpha_{D} \nabla Z\right\}-\left\{D \alpha_{D}\right\} \nabla\{Z\}\right)\right]} \\
& \text { SGS Diffusivity } 1 \quad \text { SGS Diffusivity } 2 \\
& \underbrace{+2\left[\bar{\rho}\left\{D \alpha_{D} \nabla Z\right\}-\bar{\rho}\left\{D \alpha_{D}\right\} \nabla\{Z\}\right] \cdot \nabla\{Z\}} \underbrace{-2 \bar{\rho}\left[\left\{D \alpha_{D} \nabla Z \cdot \nabla Z\right\}-\left\{D \alpha_{D}\right\}\{\nabla Z \cdot \nabla Z\}\right]} \\
& \text { SGS Diffusivity } 3 \\
& \text { SGS Diffusivity } 4 \\
& \underbrace{+2 \nabla \cdot\left[\bar{\rho}\left(\left\{\alpha_{B K}(1-Z) Z^{2} \frac{D}{T} \nabla T\right\}-\left\{\alpha_{B K}(1-Z) Z \frac{D}{T} \nabla T\right\}\{Z\}\right)\right]} \\
& \text { SGS Soret } 1 \\
& \underbrace{-2 \bar{\rho}\left[\left\{\alpha_{B K}(1-Z) Z \frac{D}{T} \nabla T \cdot \nabla Z\right\}-\left\{\alpha_{B K}(1-Z) Z \frac{D}{T} \nabla T\right\} \cdot \nabla\{Z\}\right]} \\
& \text { SGS Soret } 2 \\
& \underbrace{+2 \nabla \cdot\left[\bar{\rho}\left(\left\{D \frac{(1-Z) Z^{2}}{R_{u} T} \frac{m_{1} m_{2}}{m} \Lambda \nabla p\right\}-\left\{D \frac{(1-Z) Z}{R_{u} T} \frac{m_{1} m_{2}}{m} \Lambda \nabla p\right\}\{Z\}\right)\right]} \\
& \text { SGS Pressure } 1 \\
& \underbrace{-2 \bar{\rho}\left[\left\{D \frac{(1-Z) Z}{R_{u} T} \frac{m_{1} m_{2}}{m} \Lambda \nabla p \cdot \nabla Z\right\}-\left\{D \frac{(1-Z) Z}{R_{u} T} \frac{m_{1} m_{2}}{m} \Lambda \nabla p\right\} \cdot \nabla\{Z\}\right]},
\end{aligned}
$$

SGS Pressure 2

where term 5 in Eq. (4) was split into the classical turbulentflux form and SGS production, the turbulent fluxes were combined and only SGS-type terms emphasized. The meaning of the new SGS terms is not obvious and must be investigated.
It will be shown in Sec. VI A that the additional SGS terms in Eq. (4) have significant activity and thus are important to model. Moreover, the root mean square (rms) activity and the mean values of the SGS terms in Eq. (9) will be examined to understand their influence on the SGS variance 
evolution. Models for the new SGS terms in either Eq. (4) or Eq. (9) are not currently available, a fact which motivated investigating whether the conserved scalar conforms to a PDF having a known mathematical expression. If this is the case, the PDF could be used in a presumed-PDF approach, and therefore the SGS scalar variance could be useful for constructing the PDF, a fact which would motivate finding a model for it. The investigation of the SGS PDF shape is described next. In the remaining part of this study, we revert to the usual notation, $\widetilde{Z}$, for the Favre filtered quantities.

\section{THE PDF OF THE CONSERVED SCALAR}

The scalar statistics is the important information desired for flamelet modeling (e.g., Ref. 14). However, if the PDF is complex, it has the drawback that it can only be approximately reconstructed by a large number of its moments. ${ }^{15}$ It is certainly computationally easier if it can be shown that the PDF of the scalar conforms to a PDF having a simple mathematical form for which only a small number of moments is necessary for its reconstruction. Such simple PDFs are, for example, the Dirac, the Gaussian, and the $\beta$ functions for which one needs a maximum number of two moments for reconstruction. As stated above, the first moment of the local (SGS) PDF is always computable from LES, so that the focus is on computing the second moment, i.e., the SGS scalar variance. An extensive literature exists on the topic of the SGS scalar variance computation ${ }^{2,4,5,7,16,17}$ but not for supercritical turbulent flows for which, as seen in Sec. II, the variance equation is considerably more complex than in the already studied flows, indicating possible departures from previous findings, as implied by previous results. ${ }^{8}$ To inquire about the form of the SGS PDF, we recall here its definition and discuss a practical way to calculate it under compressible conditions.

The instantaneous SGS PDF $\left(f_{\text {sgs }}\right)$ of a scalar quantity $Z$ may be defined using the fine-grained density $\gamma[\xi, Z(\mathbf{x})]$ $\equiv \delta[Z(\mathbf{x})-\xi]$, by weighting it with the filter function $\bar{G}$ (Refs. 18 and 19) as

$$
f_{\text {sgs }}(\xi ; \mathbf{x})=\int_{\Omega} \delta\left[Z\left(\mathbf{x}^{\prime}\right)-\xi\right] \bar{G}\left(\mathbf{x}-\mathbf{x}^{\prime}\right) d \mathbf{x}^{\prime},
$$

where $\delta$ is the Dirac delta function and $\xi$ is the scalar-space variable representing $Z$. If $\bar{G}$ is a positive filter kernel, $f_{s g s}$ has the property of a PDF. As every PDF, it is constrained by the normalization condition stating that the integral over the scalar space is unity. For $Z \in[0,1]$, the instantaneous filtered value of any quantity $g(Z(\mathbf{x}))$ may be obtained by integration over the scalar space as ${ }^{17}$

$$
\bar{g}(\mathbf{x})=\int_{0}^{1} g(\xi) f_{\text {sgs }}(\xi ; \mathbf{x}) d \xi .
$$

In the same spirit, for obtaining the Favre-filtered value of every quantity $g(Z(\mathbf{x}))$ by using a PDF, a mass-weighted SGS PDF must be defined ${ }^{20}$

$$
f_{s g s_{c}}(\xi ; \mathbf{x})=\frac{1}{\bar{\rho}(\mathbf{x})} \int_{\Omega} \rho\left(\mathbf{x}^{\prime}\right) \delta\left[Z\left(\mathbf{x}^{\prime}\right)-\xi\right] \bar{G}\left(\mathbf{x}-\mathbf{x}^{\prime}\right) d \mathbf{x}^{\prime}
$$

where the subscript $c$ denotes the compressible situation, and then

$$
\tilde{g}(\mathbf{x})=\int_{0}^{1} g(\xi) f_{s g s_{c}}(\xi ; \mathbf{x}) d \xi .
$$

The mass-weighted SGS PDF (referred to as filtered mass density function (FMDF)) is defined by Jaberi et $a .^{21}$ and Raman et $a l^{22}$ in a probabilistic manner using the property that the integral of $f_{s g s_{c}}$ over the sample space gives the filtered density value. This definition is then used in the framework of a PDF approach where a transport equation for the PDF is solved. Here, instead, a PDF normalized by $\bar{\rho}(\mathbf{x})$, i.e., Eq. (12), is needed as we wish to satisfy the classical definition of the PDF integrating to unity. The SGS PDF, as defined by Eqs. (10) and (12), is not a statistical quantity as it is a one-point distribution conditioned to a given flow realization. Following Jiménez et al., ${ }^{3}$ Fox $^{23}$ and Pitsch, ${ }^{24}$ an appropriate SGS PDF utilizable for modeling purposes should instead be understood as a statistical quantity arising from a statistical sample of equivalent grid elements. Since in the a priori analysis using the DNS database the exact statistical quantity is not available, we will instead use, as an approximation of the statistical SGS PDF for evaluating the accuracy of models, statistics computed employing the onepoint SGS PDF defined above. The expectation of this PDF over homogeneous directions and the conditional (on moments) expectation are used to assess presumed PDF shapes against the DNS-extracted ones, as described below.

Assuming the filter width to be smaller than the variation scale of mean quantities and that filtering and averaging operators commute, a Favre mean (i.e., expectation) of the filtered local value may be obtained

$$
<\tilde{g}(\mathbf{x})>_{c}=\int_{0}^{1} g(\xi)<f_{s g s_{c}}(\xi ; \mathbf{x})>_{c} d \xi,
$$

where the operator $\langle\cdot\rangle_{c}$ denotes planar averages weighted by the filtered density. The quantity $\left\langle f_{s g s_{c}}\right\rangle_{c}$ is equivalent to a filtered PDF of the scalar as defined by Jiménez et al. ${ }^{3}$ for incompressible flows and it is given by the mass-weighted PDF of the scalar over planes of height equal to the filter width (coarsened-grid planes). The filtered PDF may be computed as

$$
<f_{s g s_{c}}\left(\xi ; \tilde{\xi}, \sigma_{\xi}\right)>_{c}=\int f_{c}\left(\tilde{\xi}, \sigma_{\xi}\right) f_{s g s_{c}}\left(\xi ; \tilde{\xi}, \sigma_{\xi}\right) d \tilde{\xi} d \sigma_{\xi}
$$

by using the mass-weighted joint-moment $\operatorname{PDF} f_{c}$ in conjunction with the PDF $f_{s g s_{c}}$, defined as a function of couples of moments $\left(\tilde{\xi}, \sigma_{\xi}\right)$ instead of $\mathbf{x}$ where $\sigma_{\xi}$ is the moment-space variable representing $\sigma_{Z}$, and integrating over the LESmoment space $\left(\tilde{\xi}, \sigma_{\xi}\right)$. The definition of Eq. (15) may be computationally practical since any assumed PDF model may be evaluated by replacing in Eq. (15) the presumed PDF, $f_{s g s_{\mathcal{C}}}\left(\xi ; \tilde{\xi}, \sigma_{\xi}\right)$, computed from any couple of mapped values $\left(\xi, \sigma_{\xi}\right)$, and $f_{c}\left(\tilde{\xi}, \sigma_{\xi}\right)$ as obtained from the filtered DNS database over homogeneous (coarsened-grid) directions. The $f_{s g s_{c}}$ expectation, $\left\langle f_{s g s_{c}}\right\rangle_{c}$, is used to evaluate the presumed-PDF shapes against the DNS-extracted ones.

To approximate the statistical SGS PDF, we also consider as an alternate to the above method, the optimal estimator 
method of Moreau et $a .^{25}$ which uses the conditional expectation. The latter is obtained by averaging the PDF $f_{s g s_{c}}\left(\xi ; \tilde{\xi}, \sigma_{\xi}\right)$, over a sample of PDFs having the same couples of exact moments $\left(\tilde{\xi}, \sigma_{\xi}\right)$. Practically, the computation is performed by averaging over SGS volumes having couples of moments $\left(\tilde{\xi}, \sigma_{\xi}\right)$ very close to a selected one, according with a fixed standard deviation chosen such as to have accurate statistics. The conditional expectation of the DNS-extracted quantity $f_{s g s_{c}}\left(\xi ; \tilde{\xi}, \sigma_{\xi}\right)$ is then compared to the assumed PDFs computed using the exact moments. Here, the Favre-conditional expectation is obtained by mass-weighted averaging using the filtered local density, and statistics are computed over a slab of the mixing layer in order to increase the sample size.

In this study, three local PDFs will be assessed: the $\beta$ PDF, the Gaussian, and the Dirac ones. These PDFs are here briefly recalled:

- The $\beta$ PDF:

$$
f_{s g s_{c}}\left(\xi ; \alpha\left(\tilde{\xi}, \sigma_{\xi}\right), \beta\left(\tilde{\xi}, \sigma_{\xi}\right)\right)=\frac{\Gamma(\alpha+\beta)}{\Gamma(\alpha) \Gamma(\beta)} \xi^{\alpha-1}(1-\xi)^{\beta-1},
$$

where $\Gamma$ is the Gamma function and parameters $\alpha$ and $\beta$ are defined by

$$
\alpha=\tilde{\xi}\left(\frac{\tilde{\xi}(1-\tilde{\xi})}{\sigma_{\xi}}-1\right), \quad \beta=(1-\tilde{\xi})\left(\frac{\tilde{\xi}(1-\tilde{\xi})}{\sigma_{\xi}}-1\right) .
$$

- The Gaussian PDF:

$$
f_{s g s_{c}}\left(\xi ; \tilde{\xi}, \sigma_{\xi}\right)=\frac{\frac{1}{\sqrt{2 \pi \sigma_{\xi}}} e^{-\frac{(\xi-\xi \xi)^{2}}{2 \sigma_{\xi}}}}{\frac{1}{2}\left(1+\operatorname{erf} \frac{b-\tilde{\xi}}{\sqrt{2 \sigma_{\xi}}}\right)-\frac{1}{2}\left(1+\operatorname{erf} \frac{a-\tilde{\xi}}{\sqrt{2 \sigma_{\xi}}}\right)} .
$$

As the scalar $\xi$ is bounded between 0 and 1 , the Gaussian distribution is truncated and the resulting PDF is thus renormalized using the difference between the cumulative distribution functions evaluated at $b=1$ and $a=0$. This computed distribution has no longer the same mean and variance as the original distribution. As an alternate, a clipped Gaussian is also considered. ${ }^{26}$

- The Dirac PDF:

$$
f_{s g s_{c}}(\xi ; \tilde{\xi})=\delta(\xi-\tilde{\xi}) .
$$

The Dirac PDF is utilized when the scalar $\xi$ is only modeled through its mean, $\tilde{\xi}$.

In Sec. VI B we present results from computations with these PDFs using both methods to approximate the statistical SGS PDF, and particularly we show that whereas the Dirac and Gaussian PDF are generally deficient, the $\beta$ PDF typically yields a good approximation, a fact which provided the incentive to directly model the scalar variance, as described in the following.

\section{DIRECT MODELING OF THE SGS SCALAR VARIANCE}

Two types of models appear to be promising candidates for modeling small-scale effects removed through filtering. The first model is of a structural type $\mathrm{e}^{27}$ based on the approximate deconvolution model (ADM) ${ }^{28}$ while the second one is of a functional type using a gradient-based scaling law. ${ }^{4}$ The two models are conceptually different as the first one arises from a mathematical derivation with no assumption regarding the nature of scale interactions, while the second one uses a mixing-length hypothesis in conjunction with an equilibrium assumption. The original contribution of our work is the investigation of the ADM capability for computing $\sigma_{Z}$ in variable density flows, and the extension of a recent dynamic gradient-based formulation ${ }^{5}$ to compressible conditions for modeling $\sigma_{Z}$.

\section{A. The approximate deconvolution model}

The deconvolution procedure ${ }^{28}$ relies on the assumption that there exists an inverse operator $\bar{G}^{-1}=\sum_{l=0}^{\infty}(I-\bar{G})^{l}$ such as

$$
Z^{*}=\left[\sum_{l=0}^{N}(I-\bar{G})^{l}\right] * \bar{Z},
$$

where $I$ is the identity operator and $Z^{*}$ represents an approximation of the original field $Z$, on the LES mesh grid, at the series truncation order $N$. The series is known to converge for $\|I-\bar{G}\|<1$, and written using de van Cittert method ${ }^{29}$ based on the Neumann series leads to

$$
Z^{*}=\bar{Z}+(\bar{Z}-\overline{\bar{Z}})+(\bar{Z}-2 \overline{\bar{Z}}+\overline{\bar{Z}})+\ldots,
$$

where the accuracy of $Z^{*}$ depends on $N$. In practice, due to the numerical discretization (projection on a coarse mesh grid), only the recoverable part of the original field $Z$ may be obtained by a deconvolution procedure ${ }^{30}$ Furthermore, the deconvolution relies on the form of the convolution-filter kernel and on the shape of the spectrum of the field. ${ }^{17}$

The series' rate of convergence is situation dependent. For example, it has been found ${ }^{31}$ that $N=3$ is sufficient to bring an improvement in that for a quantity $\phi,\left(\phi^{*}-\bar{\phi}\right)$ is not null showing that the expansion is indeed effective, and for $N \geq 5$ the value of $\left(\phi^{*}-\bar{\phi}\right)$ did not change appreciably from that obtained with $N<5$. In other studies specifically directed at $Z$, it was found that the series converges very slowly. Pantano and Sarkar ${ }^{16}$ tested the deconvolution procedure in an a priori analysis using the DNS of a temporal mixing layer; they showed that even with $N=5$ (i.e., fourthorder approximation) and a small filter size $\left(\bar{\Delta} / \Delta x_{D N S}=4\right.$, where $\Delta x_{D N S}$ is the grid spacing for a simulation where all scales relevant to most of the dissipation are resolved, as in DNS), no more than $88 \%$ of the total SGS scalar-variance amount (in the peak zone) was recovered. The rate of convergence of the series and the recoverable amount of the field are thus the main issues of the method. In order to recover all effects of the smallest scales, Stolz et al. ${ }^{28}$ suggested a 
secondary filtering through the use of a relaxation parameter in the Navier-Stokes (NS) equations. To mitigate both these issues, Pantano and Sarkar ${ }^{16}$ and Mellado et al. ${ }^{17}$ proposed an approximate reconstruction using moments (ARM) method which, based on the definition of an intermediate field, leads to computing the SGS scalar variance by imposing equality between the real moment and that of the intermediate field. This procedure results in an expression for a parameter $c_{0}$, characterizing the model, that is obtained by finding the real and positive solution of a second-order polynomial equation. The polynomial coefficients $a_{0}, a_{1}, a_{2}$ are computed using the presumed shape of the spectrum of the scalar quantity. Once the shape of the spectrum is selected, $c_{0}$ may be precomputed and stored in a two-dimensional table of coordinates represented by two dimensionless quantities: the Péclet number and the ratio between the filter width and a large scale of the scalar fluctuations. That approach was developed for incompressible flows. Under compressible conditions, that model requires rewriting for the conservative quantities for which the governing equations are solved in LES of compressible flows; but then, equality between moments would not lead to an explicit expression for the SGS scalar variance unless additional assumptions are invoked. Moreover, when in presence of variable density, the shape of the spectrum cannot be built using calibrated constants computed either by using the Reynolds-averaged scalar variance or by employing the Favre-averaged one. This is why, considering the practical utilization in LES, we opt to explore a classical deconvolution procedure.

Since in LES it is the conservative rather than primitive quantities which are calculated, we are legitimately inquiring as to whether the SGS scalar variance could be accurately reconstructed employing a deconvolution procedure for these quantities. Indeed, for reconstructing the approximated scalar field and building the SGS (Favre) scalar variance, we must reconstruct $\rho$ and $Z$ through

$$
\begin{gathered}
\rho^{*}=\bar{\rho}+(\bar{\rho}-\overline{\bar{\rho}})+(\bar{\rho}-2 \overline{\bar{\rho}}+\overline{\bar{\rho}})+\ldots, \\
(\rho Z)^{*}=\overline{\rho Z}+(\overline{\rho Z}-\overline{\overline{\rho Z}})+(\overline{\rho Z}-2 \overline{\overline{\rho Z}}+\overline{\overline{\rho Z}})+\ldots,
\end{gathered}
$$

which then permits writing

$$
Z^{* *}=(\rho Z)^{*} / \rho^{*},
$$

invoking the assumption

$$
Z^{* *} \simeq Z^{*}
$$

Equation (25) implies equality between second-order moments of $Z^{* *}$ and $Z^{*}$. The nonlinear function of the approximate field, the Favre SGS scalar variance, is then built, for consistency, ${ }^{16}$ by using the deconvoluted fields as

$$
\sigma_{Z}=\frac{\overline{\rho^{*} Z^{* *} Z^{* *}}}{\overline{\rho^{*}}}-\frac{\overline{\rho^{*} Z^{* *}}}{\overline{\rho^{*}}} \frac{\overline{\rho^{*} Z^{* *}}}{\overline{\rho^{*}}} .
$$

The assumption of Eq. (25) is exact if both $\rho$ and $\rho Z$ fields are totally recovered and an infinite expansion is used. Otherwise, there is no proof that ratio between series (23) and
(22) converges to the recoverable part of the quantity $Z$, which is $Z^{*}$ at a given truncation order $N$. Such an assumption, although not trivial, has already been used by Stolz et al. ${ }^{32}$ while reconstructing flux terms of the compressible NS equations and by Dubois et al. ${ }^{33}$ in the first of a two-step procedure for estimating the SGS stress tensor. The difference between the previous studies and our investigation is that ADM is here used in the context of a soft-deconvolution problem ${ }^{27}$ in which no additional models for recovering the deficient SGS part are provided. In Sec. VI C 1 we evaluate the assumption of Eq. (25) and the equality between moments of $Z^{* *}$ and $Z^{*}$ for several truncation orders. We also assess the ADM model using the series Eqs. (22) and (23), with, consistently, both series truncated at same order.

\section{B. The dynamic model}

A Smagorinsky-type model for predicting the SGS scalar variance of compressible flows was proposed by Pierce and Moin ${ }^{4}$ using a dynamic procedure. ${ }^{34}$ This model was similar to that of Moin et al. ${ }^{35}$ for modeling the SGS stress tensor and heat flux under compressible conditions. The model of Pierce and Moin $^{4}$ has been extensively used over the years ${ }^{7,20,36,37}$ as an alternate to the scale-similarity model suggested by Cook and Riley ${ }^{2}$ based on the idea of Bardina et al. ${ }^{38}$ The scale-similarity model has been explored in several different configurations. ${ }^{3,7,39,40}$ Recently, Balarac et al., ${ }^{5}$ using the optimal estimator concept of Moreau et al., ${ }^{25}$ showed that the irreducible error associated with a Smagorinsky-type model is relatively small if compared to that evaluated for a scalesimilarity model, meaning that the functional form of a gradient-based model has significant potential in LES for reproducing the SGS scalar variance. This has also been observed by Wall et al. ${ }^{36}$ when studying the performance of the two models used in that study in conjunction with a presumed PDF approach. On the other hand, Balarac et al. ${ }^{5}$ showed that the quadratic error associated with the gradient-based model notably increases with filter width when a classical dynamic procedure is used for computing the model coefficient. In the present study, the Balarac et al. ${ }^{5}$ model, which is for incompressible flow, is reformulated under compressible conditions and evaluated for compressible supercritical turbulent mixing layers in Sec. VI C 2.

For completeness, the Pierce and Moin ${ }^{4}$ dynamic model is briefly recalled and for consistency we use their notation, where $\widehat{()}$ is the unweighted test filter at test-filter level $\widehat{G}$ corresponding to the filter width $\widehat{\Delta}$ and

$$
\widetilde{Z}=\widehat{\bar{\rho} \widetilde{Z}} / \hat{\bar{\rho}} \text {. }
$$

In the classical dynamic model, ${ }^{4}$ first the variance is related to the gradient of the resolved scalar field as

$$
\bar{\rho} \sigma_{Z}=\bar{\rho}(\widetilde{Z Z}-\widetilde{Z} \widetilde{Z})=C_{d} \bar{\Delta}^{2} \bar{\rho}|\nabla \widetilde{Z}|^{2},
$$

which, when filtered, yields the following expression:

$$
\widehat{\bar{\rho} \widetilde{Z}}-\widehat{\bar{\rho} \widetilde{Z} \widetilde{Z}}=C_{d}^{\prime} \bar{\Delta}^{2}\left(\widehat{\bar{\rho}|\nabla \widetilde{Z}|^{2}}\right) \text {. }
$$


A similar model is then proposed at the filter level corresponding to the convolution of $\bar{G}$ and $\widehat{G}$, i.e., $\widehat{\bar{G}}=\bar{G} * \widehat{G}$, associated to the filter width $\widehat{\bar{\Delta}}$

$$
\widehat{\bar{\rho} \overline{Z Z}}-\widehat{\bar{\rho}} \widetilde{Z} \widetilde{Z}=C_{d}^{\prime \prime} \widehat{\bar{\Delta}}^{2} \widehat{\bar{\rho}}|\nabla \widetilde{Z}|^{2}
$$

Finally, subtracting Eq. (29) from Eq. (30) and assuming $C_{d}^{\prime}=C_{d}^{\prime \prime}=C_{d}$ (implying either that the flow variation is such that filtering with the grid or test filter produces analogous fields or that the coefficients have a slow variation) yields

$$
\widehat{\bar{\rho} \widetilde{Z} \widetilde{Z}}-\widehat{\bar{\rho}} \widetilde{Z} \widetilde{Z}=C_{d}\left[\hat{\bar{\Delta}}^{2} \widehat{\bar{\rho}}|\nabla \widetilde{Z}|^{2}-\bar{\Delta}^{2}\left(\widehat{\bar{\rho}|\nabla \widetilde{Z}|^{2}}\right)\right],
$$

which may be generically written as

$$
\mathcal{L}=C_{d} \mathcal{M}
$$

where $\mathcal{L} \equiv(\widehat{\bar{\rho} \widetilde{Z} \widetilde{Z}}-\widehat{\bar{\rho}} \widetilde{Z} \widetilde{Z})$ is the generalized Leonard term and $\mathcal{M}$ is defined as

$$
\mathcal{M}=\widehat{\bar{\Delta}}^{2} \widehat{\bar{\rho}}|\nabla \widetilde{Z}|^{2}-\bar{\Delta}^{2}\left(\widehat{\bar{\rho}|\nabla \widetilde{Z}|^{2}}\right) .
$$

Since both $\mathcal{L}$ and $\mathcal{M}$ are computable from the LES solution, $C_{d}$ may be dynamically computed over homogeneous directions either as the ratio between averaged $\mathcal{L}$ and $\mathcal{M}$ or through the least-square averaging $\left(\right.$ Lilly $\left.^{41}\right)$ which optimizes the local value by minimizing the quadratic error. This dynamic procedure uses a compressible version of the Germano identity, ${ }^{42}$ originally written for the SGS stress tensor ${ }^{35,43}$

$$
L_{i j}=\widehat{\bar{\rho}} T_{i j}-\widehat{\bar{\rho} \tau_{i j}}
$$

where

$$
\widehat{\bar{\rho}} T_{i j} \equiv \widehat{\bar{\rho} \widehat{u_{i} u_{j}}}-\widehat{\bar{\rho}} \breve{\tilde{u}_{i}} \breve{u_{j}}, \quad \widehat{\bar{\rho} \tau_{i j}} \equiv\left(\widehat{\bar{\rho} \widehat{u_{i} u_{j}}}\right)-\left(\widehat{\bar{\rho} \widetilde{u_{i}} \widetilde{u_{j}}}\right) .
$$

When Eq. (34) is adapted to the scalar variance, it may be written as

$$
L=\widehat{\bar{\rho}} \Sigma_{Z}-\widehat{\bar{\rho} \sigma_{Z}}
$$

where

$$
\widehat{\bar{\rho}} \Sigma_{Z} \equiv(\widehat{\bar{\rho} \widetilde{Z Z}}-\widehat{\bar{\rho}} \widetilde{Z Z})
$$

Equation (30) was questioned by Balarac et al. ${ }^{5}$ who emphasized that in the classical model ${ }^{4}$ a generic filtered quantity $\hat{\bar{f}}$ is not directly obtained through a single convolution but rather from two sequential filterings. Using the Bedford and $\mathrm{Yeo}^{44}$ expansion (which provides a power series for the nonlinear filtered generic term $\overline{f g}$ as a function of the resolved quantities $\bar{f}$ and $\bar{g}$ ) applied twice to the filtered quantities, Balarac et $a l{ }^{5}$ pointed out a missing leading-order term in Eq. (30) and provided an alternative model for $\mathcal{M}$ that involves the use of the Leonard term expansion. The same reasoning is possible for compressible flows using an appropriate Taylor expansion. Such an approximate expansion was suggested by Vreman ${ }^{43}$ for modeling the SGS stress tensor and is adapted here for isotropic filter to the scalar variance as

$$
\begin{aligned}
\bar{\rho} \sigma_{Z}= & \overline{\rho Z Z}-\overline{\rho Z} \overline{\rho Z} / \bar{\rho} \\
= & \rho Z Z+\frac{\bar{\Delta}^{2}}{24} \nabla^{2}(\rho Z Z)-\left(\rho Z+\frac{\bar{\Delta}^{2}}{24} \nabla^{2}(\rho Z)\right) \\
& \times\left(\rho Z+\frac{\bar{\Delta}^{2}}{24} \nabla^{2}(\rho Z)\right) /\left(\rho+\frac{\bar{\Delta}^{2}}{24} \nabla^{2} \rho\right)+O\left(\bar{\Delta}^{4}\right) \\
= & \frac{\bar{\Delta}^{2}}{12} \rho|\nabla Z|^{2}+O\left(\bar{\Delta}^{4}\right)
\end{aligned}
$$

where the approximation

$$
\left(\rho+\frac{\bar{\Delta}^{2}}{24} \nabla^{2} \rho\right)^{-1}=\frac{1}{\rho}-\frac{\bar{\Delta}^{2}}{24 \rho^{2}} \nabla^{2} \rho+O\left(\bar{\Delta}^{4}\right)
$$

has been made to obtain the final result. A similar expansion

$$
\rho=\bar{\rho}+O\left(\bar{\Delta}^{2}\right), \quad \mathrm{Z}=\widetilde{\mathrm{Z}}+O\left(\bar{\Delta}^{2}\right)
$$

used in Eq. (38) leads to

$$
\bar{\rho} \widetilde{Z Z}=\bar{\rho} \widetilde{Z} \widetilde{Z}+\frac{\bar{\Delta}^{2}}{12} \bar{\rho}|\nabla \widetilde{Z}|^{2}+O\left(\bar{\Delta}^{4}\right) .
$$

Equation (41) is conceptually similar to that used by Balarac et $a l .^{5}$ for incompressible conditions, and in the same way it may be used for obtaining $\widehat{\bar{\rho}} \Sigma_{Z}$, i.e., the left hand side (1.h.s.) term of Eq. (30). First, the test filter $\widehat{G}$, is applied to Eq. (41)

$$
\widehat{\bar{\rho} \widetilde{Z Z}}=\widehat{\bar{\rho} \widetilde{Z} \widetilde{Z}}+\frac{\bar{\Delta}^{2}}{12}\left(\widehat{\bar{\rho}|\nabla \widetilde{Z}|^{2}}\right)+O\left(\bar{\Delta}^{4}\right),
$$

then the first term on the r.h.s. is expanded at the filter level $\hat{G}$ based on $\bar{\rho}$ and $\widetilde{Z}$ using the approximate expansion of Eqs. (38)-(40) applied to these variables, leading to

$$
\widehat{\bar{\rho} \widetilde{Z Z}}=\widehat{\bar{\rho}} \widetilde{Z} \widetilde{Z}+\frac{\hat{\Delta}^{2}}{12} \hat{\bar{\rho}}|\nabla \widetilde{Z}|^{2}+\frac{\bar{\Delta}^{2}}{12}\left(\widehat{\bar{\rho}|\nabla \widetilde{Z}|^{2}}\right)+O\left(\hat{\Delta}^{4}, \bar{\Delta}^{4}\right) .
$$

The above equation, reformulated using a constant to account for the truncation error, leads to

$$
\widehat{\bar{\rho} \widetilde{Z Z}}-\widehat{\bar{\rho}} \widetilde{Z} \widetilde{Z}=C_{d}^{\prime \prime \prime}\left[\hat{\Delta}^{2} \hat{\bar{\rho}}|\nabla \widetilde{Z}|^{2}+\bar{\Delta}^{2}\left(\widehat{\bar{\rho}|\nabla \widetilde{Z}|^{2}}\right)\right]
$$

which represents another form for the 1.h.s. term in Eq. (30). Subtracting Eq. (29) from Eq. (44) and assuming $C_{d}^{\prime}=C_{d}^{\prime \prime \prime}=C_{d}$ (under the same implications as when adopting $C_{d}^{\prime}=C_{d}^{\prime \prime}=C_{d}$ ) leads to a new formulation of $\mathcal{M}, \mathcal{M}_{n}$

$$
\mathcal{M}_{n}=\hat{\Delta}^{2} \hat{\bar{\rho}}|\nabla \widetilde{\widetilde{Z}}|^{2}
$$

representing the leading order of the Leonard term expansion when $C_{d}$ palliates for the truncated terms in the Taylor series 
expansion. Balarac et al. $^{5}$ pointed out that Eq. (43) contains a new leading order term with respect to Eq. (30) which, when taken into account, yields the new formulation of $\mathcal{M}$, $\mathcal{M}_{n}$. Although we obtain the same result as Balarac et al., ${ }^{5}$ $\mathcal{M}_{n}$ for compressible conditions, Eq. (30) is different from the corresponding equation of Balarac et al. ${ }^{5}$ as far as filter width used. Balarac et al..$^{5}$ did not discuss the disparity between the filter width used in the classical formulation and the filter width needed in the Leonard term expansion, and it appears that the same filter width was used. However, when the correct filter width is used, the issue of the new leading order term in the formulation of Eq. (30) is moot, and the result is that only an alternative formulation for $\widehat{\bar{\rho}} \Sigma_{Z}$ is derived. According to Eq. (45), it is clear that the new formulation uses $\hat{\Delta}$. For the classical formulation, according to Eq. (33), the filter is though $\bar{\Delta}$. This finding is in agreement with the study of Brun and Friedrich. ${ }^{45}$ Using the Vreman et al. ${ }^{46}$ estimate, the filter width associated with the filter level $\bar{G}$ is

$$
\widehat{\bar{\Delta}}^{2}=\widehat{\Delta}^{2}+\bar{\Delta}^{2}
$$

representing an approximation for double top-hat filtering. In order to explore the impact of the filter width used in the classical formulation and thus to asses the correctness of the results, we present in Sec. VI C 2 an a priori analysis using the classical formulation in conjunction with each of the approximations for the filter width $\widehat{\bar{\Delta}}$ at the filter level $\widehat{\bar{G}}$ : (1) $\widehat{\bar{\Delta}}^{2}=\widehat{\Delta}^{2}+\bar{\Delta}^{2}$ (correct approximation) and (2) $\widehat{\bar{\Delta}}=\widehat{\Delta}$ (incorrect with a top-hat filter) and then compare the results from these two computations with those from the new formulation based on the Leonard term expansion for which the filter is demonstrated to be $\widehat{\Delta}$.

We can thus evaluate the gradient-based model for $\sigma_{Z}$ using two different formulations. The first formulation is the classical one employing

$$
C_{d}=\frac{\langle\mathcal{L} \mathcal{M}\rangle}{\langle\mathcal{M} \mathcal{M}\rangle},
$$

where \langle\rangle denotes averages over homogeneous planes. The second formulation, which we call the "new model," is the present one and uses

$$
C_{d}=\frac{\left\langle\mathcal{L} \mathcal{M}_{n}\right\rangle}{\left\langle\mathcal{M}_{n} \mathcal{M}_{n}\right\rangle} .
$$

Since the evaluation of the correct filter width at filter-level $\bar{G}$ represents the main issue for computing $C_{d}$ using a dynamic procedure, the model based on the Leonard term expansion represents a solution to this quandary, as shown in Sec. VI C 2.

\section{DESCRIPTION OF THE DNS DATABASE}

A complete and detailed description of the DNS database has already been provided in Refs. 8-12. Out of the complete database consisting of three sets of species heptane/nitrogen $(\mathrm{HN})$, oxygen/hydrogen $(\mathrm{OH})$, and oxygen/helium $(\mathrm{OHe})$ for which several simulations were conducted, we select here three simulations, HN600, OH750, and OHe600 for examina- tion so as to enhance the generality of the results. The DNS were conducted for a temporal mixing layer and initiated with (vorticity-thickness based) Reynolds number of 600 (HN600, OHe600) and $750(\mathrm{OH} 750)$ where $\delta_{\omega, 0}=\Delta U_{0} /\left\langle\partial u_{1} / \partial x_{2}\right\rangle$ is the initial vorticity thickness, \langle\rangle is here performed over $\left(x_{1}\right.$, $x_{3}$ ) planes, and $\Delta U_{0}$ is the initial velocity difference across the layer. In all cases, the DNS grid, $\Delta x_{D N S}$, was fine enough to resolve the scales overwhelmingly responsible for the dissipation. Transitional states were achieved for all of these layers at $t_{t r}^{*}=135$ for HN600, $t_{t r}^{*}=150$ for OH750, $t_{t r}^{*}=220$ for OHe600, where $t^{*} \equiv t \Delta U_{0} / \delta_{\omega, 0}$; the corresponding transitional Reynolds numbers were 1452, 1507, and 2004. Selle et al. ${ }^{9}$ showed that this database is relevant for fully-turbulent flow modeling.

\section{RESULTS}

In all computations, the results of which are presented below, we use a top-hat filter and a trapezoidal integration method.

\section{A. Activity of terms in the scalar variance equation}

All terms in Eqs. (4) and (9) were a priori evaluated using the three DNS realizations discussed above. The goal of this evaluation is: (1) to assess the magnitude of the filtered terms with respect to that of the resolved terms for modeling purposes (Eq. (4)) and (2) to assess the magnitude of the SGS terms with respect to that of the resolved terms in order to identify which SGS quantities are important to model (Eq. (9)). A cubic top-hat filter and several filter widths, $\bar{\Delta}$, were used in the evaluation, but only the analysis corresponding to the ratio $\bar{\Delta} / \Delta x_{D N S}=8$ is here presented, being representative of all ratio values.

Depicted in the Figure 1 is the activity of terms in Eq. (4) as measured by the rms magnitude of each term. The results indicate that terms 4 and 5, which involve the classical subgrid fluxes, dominate showing that our transitional databases have indeed the turbulent characteristics that make them relevant to this study. The importance of the advective term increases with the strength of the HDGM at transition $^{11}$ (compare OH750 with HN600) and with increasing Reynolds number value at transition ${ }^{9}$ (compare HN600 and OHe600). The diffusion term (term 1) is of same order of magnitude as the advection term only for $\mathrm{OH} 750$, because the hydrogen diffusivity is very large. Terms 2 and 3 are somewhat smaller than the advection term for HN600 and OHe600 but significantly larger for OH750. For both HN600 and OHe600 and less so for OH750, term 3 is larger than term 2, which is a manifestation of the SGS magnitude associated with the Fick-issued dissipation. Term 6, representing SGS effects, could be neglected in all cases. Evaluation of term 7, expressing the diffusivity spatial variation, shows that it is of the same order of magnitude as the resolved diffusion (term 1) for HN600 and OHe600 simulations and even larger for the OH750. Thus, term 7 is non negligible in Eq. (4) under all circumstances. Similarly, term 8 representing the effect of spatially varying diffusivity is comparable to term 1 for all cases and should be included when modeling Eq. (4), particularly when diffusion plays an 

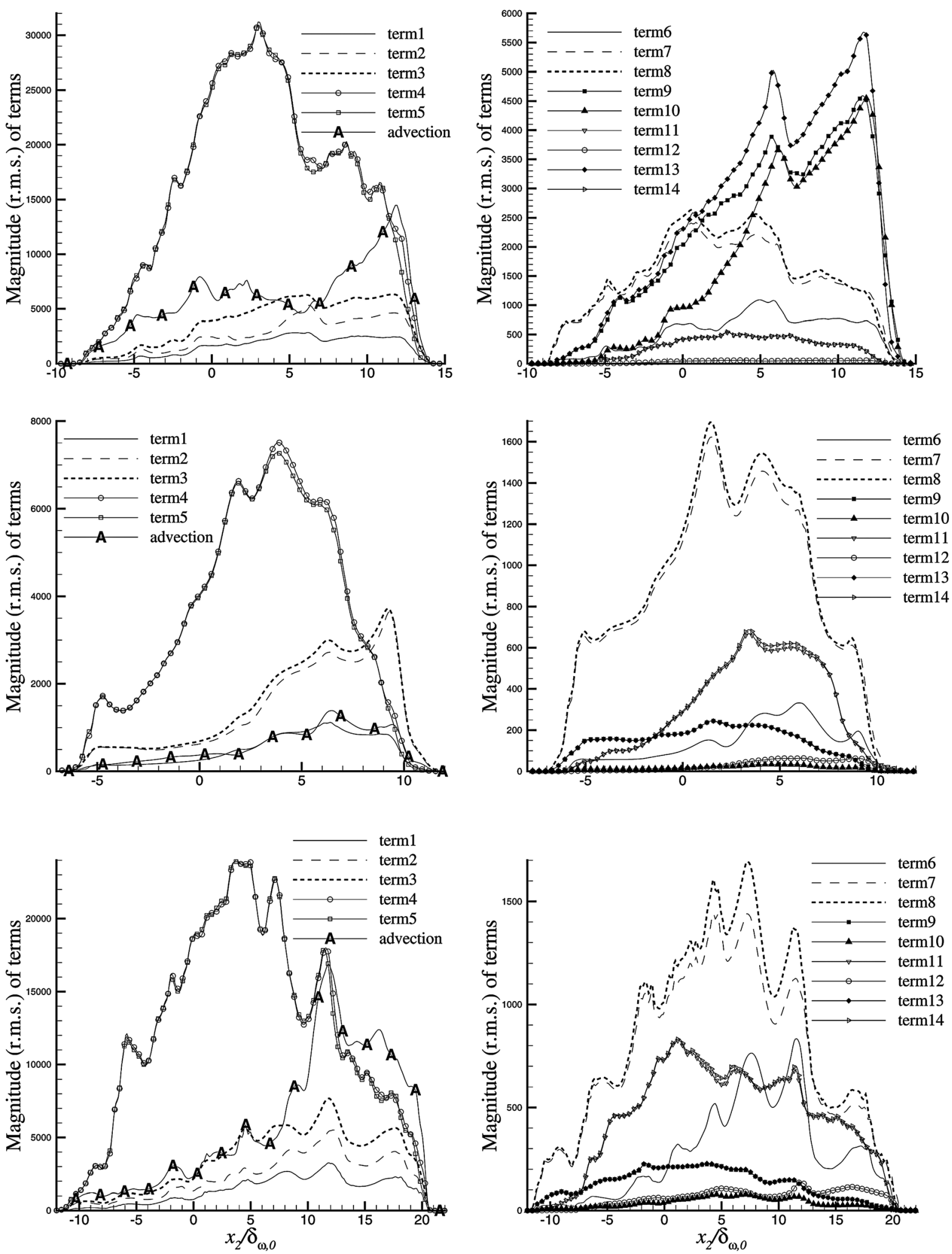

FIG. 1. Planar rms activity of terms in Eq. (4) as extracted from the filtered DNS databases at $t_{t r}^{*}$. Top: HN600; center: OH750; bottom: OHe600. $\bar{\Delta} / \Delta x_{D N S}=8$. Units are $\mathrm{kg} /\left(\mathrm{m}^{3} \mathrm{~s}\right)$.

important role during mixing (i.e., OH750). In contrast, the terms arising from filtering of the Soret contribution, i.e., terms 9, 10, and 13 are only substantial for the HN600 case, while those stemming from filtering of the term proportional to the pressure gradient, namely terms 11 and 14, only play a minor role in the $\mathrm{OH} 750$ and $\mathrm{OHe} 600$ layers and are unim- portant for the HN600 case. Term 12 is found negligible everywhere.

Figure 2 shows the results from the a priori assessment of the terms in Eq. (9). Both the planar average and the rms activity are computed in order to evaluate not only the importance but also the nature (i.e., diffusive, dissipative, etc.) 

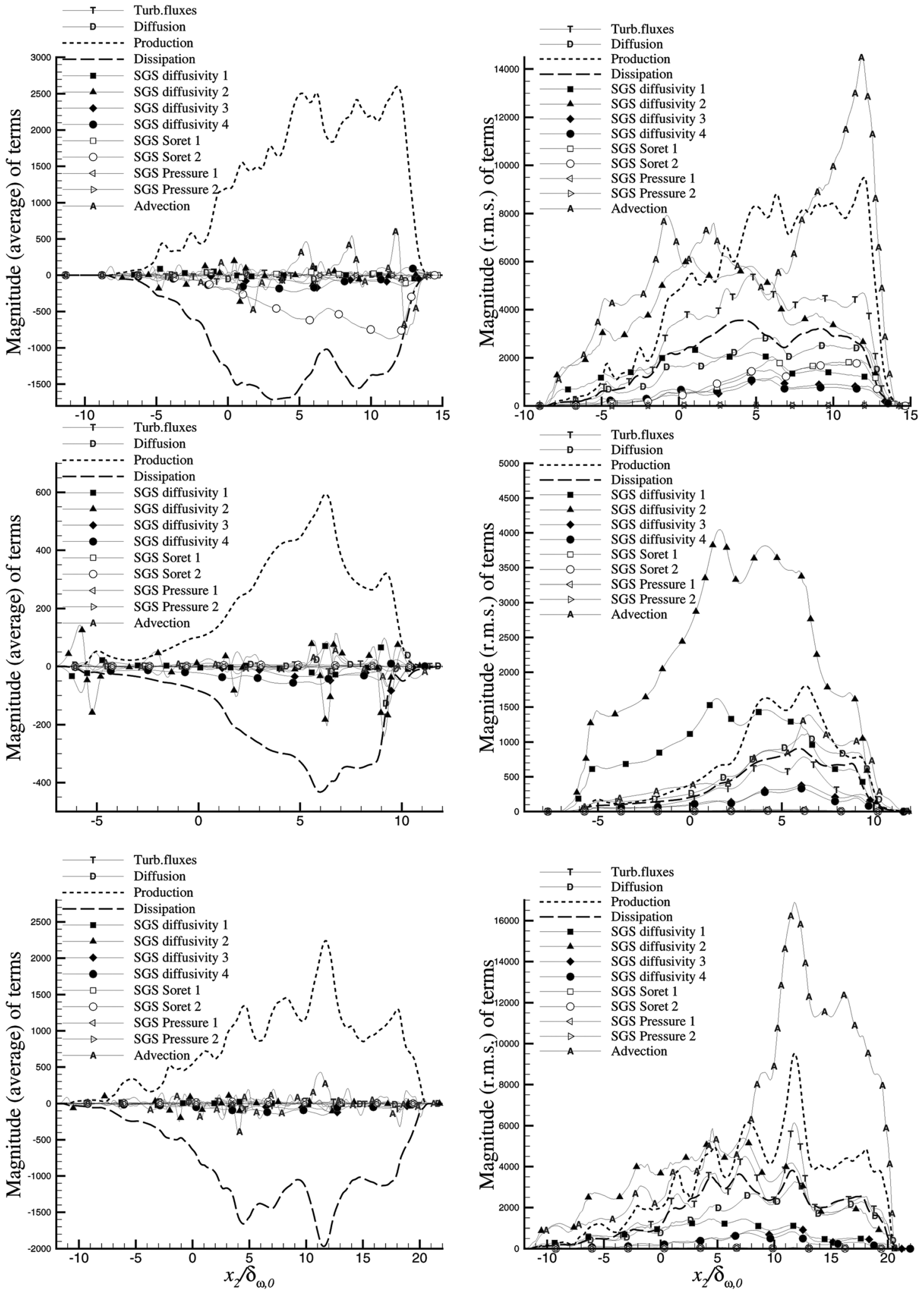

FIG. 2. Planar averages (left) and planar rms activity (right) of terms in Eq. (9). Extracted from the filtered DNS databases at $t_{t r}^{*}$. Top: HN600; center: OH750; bottom: OHe600. $\bar{\Delta} / \Delta x_{D N S}=8$. Units are $\mathrm{kg} /\left(\mathrm{m}^{3} \mathrm{~s}\right)$.

of each term. Considering the planar averages, in all cases, the magnitude of transport terms is small, and the production term is comparable in magnitude to the Fick-issued SGS dissipation term. Due to the difficulty of entraining the lower-stream heavy fluid, the mixing layer growth is moderate which explains the small value of the advection term compared to that of the SGS production and SGS Fickissued dissipation. Additionally, for the HN600 layer the term denoted by SGS Soret 2 has considerable negative magnitude, adding to the Fick-issued dissipation. This observation is consistent with the definition of the total scalar dissipation ${ }^{13}$ 

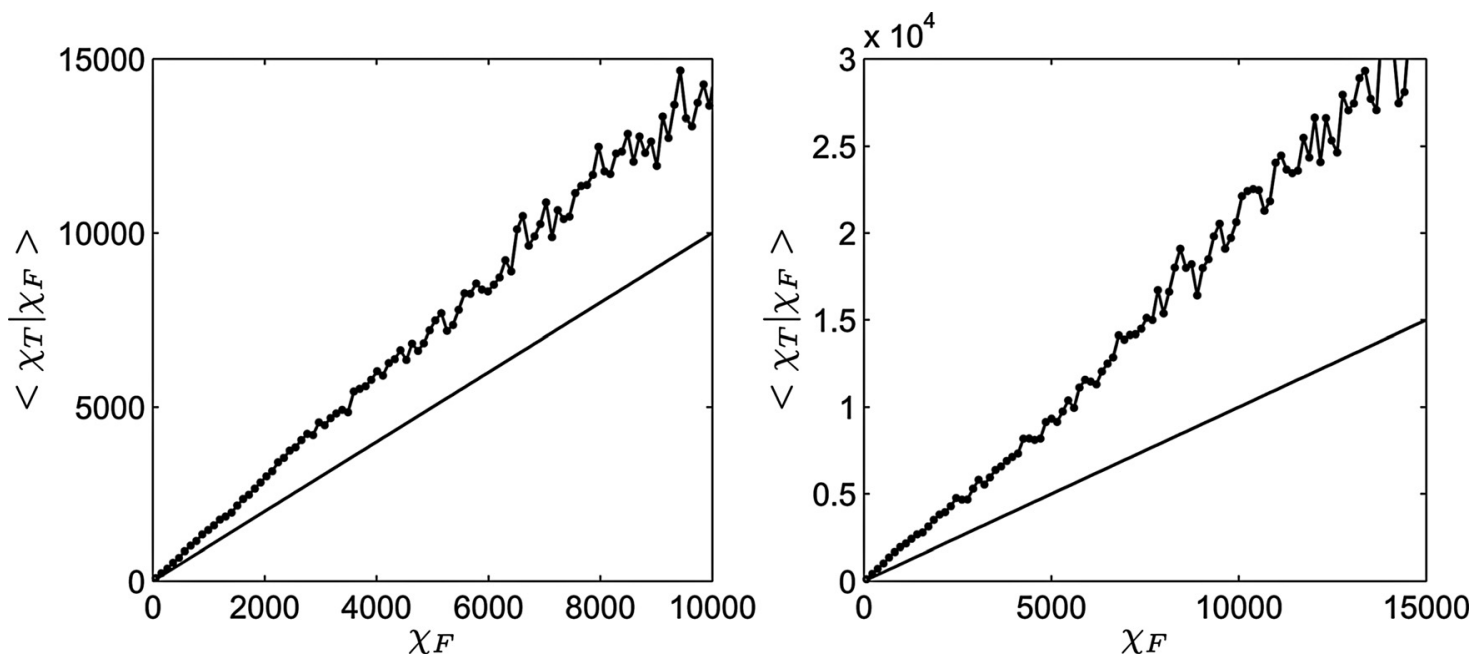

FIG. 3. Conditional average of $\chi_{T}$ on $\chi_{F}$ extracted from the HN600 DNS database over planes of coordinates $x_{2} / \delta_{\omega, 0}=0.44$ (left) and $x_{2} / \delta_{\omega, 0}=5.11$ (right) at $t_{t r}^{*}$.

$$
\chi_{T}=\frac{1}{\rho D \alpha_{D}} \mathbf{J}_{\alpha} \cdot \mathbf{J}_{\alpha}
$$

that is here contrasted to the Fick-issued dissipation

$$
\chi_{F}=\rho D \alpha_{D} \nabla Z \cdot \nabla Z,
$$

which is only one of the six terms of Eq. (49). Obviously, the Soret contribution plays an important role for the HN600
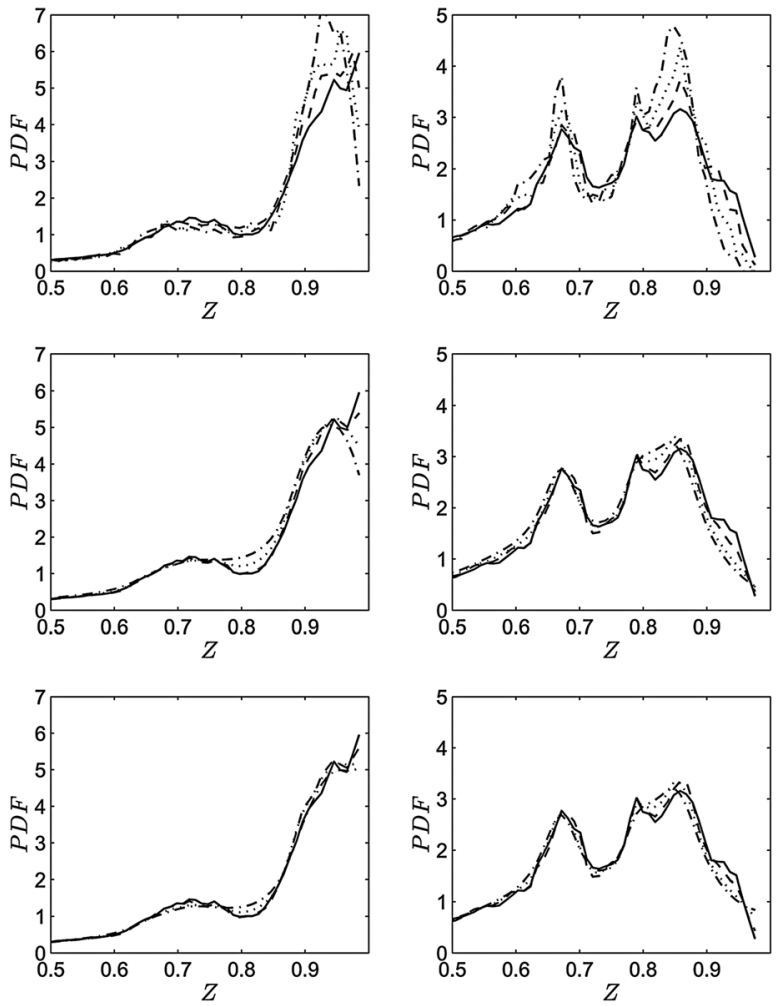

FIG. 4. Filtered PDFs $\left\langle f_{s g s_{c}}\right\rangle_{c}$, from the HN600 DNS at $t_{t r}^{*}$ over planes of coordinates $x_{2} / \delta_{\omega, 0}=0.44$ (left) and $x_{2} / \delta_{\omega, 0}=5.11$ (right). Exact SGS: solid lines. Assumed PDFs are the Dirac (top), the Gaussian (center), and the Beta (bottom) functions. $\bar{\Delta} / \Delta x_{D N S}=4$, dashed lines; $\bar{\Delta} / \Delta x_{D N S}=8$, dotted lines; $\bar{\Delta} / \Delta x_{D N S}=12$, dot-dashed lines. layer in the destruction of the scalar fluctuations at the smallest scales, but clearly its importance is binary-species dependent as it has no impact on the mixing of the oxygen with hydrogen or helium. In order to understand the behavior of $\chi_{F}$ with respect to $\chi_{T}$, we illustrate in Fig. 3 the average of $\chi_{T}$ conditioned on $\chi_{F}$ in two planes of the HN600 mixing layer $\left(x_{2} / \delta_{\omega, 0}=0.44\right.$ which is in the central part of the layer and $x_{2} / \delta_{\omega, 0}=5.11$ which away from the center but still in a significant mixing region). The results indicate that $\chi_{T}$ is larger
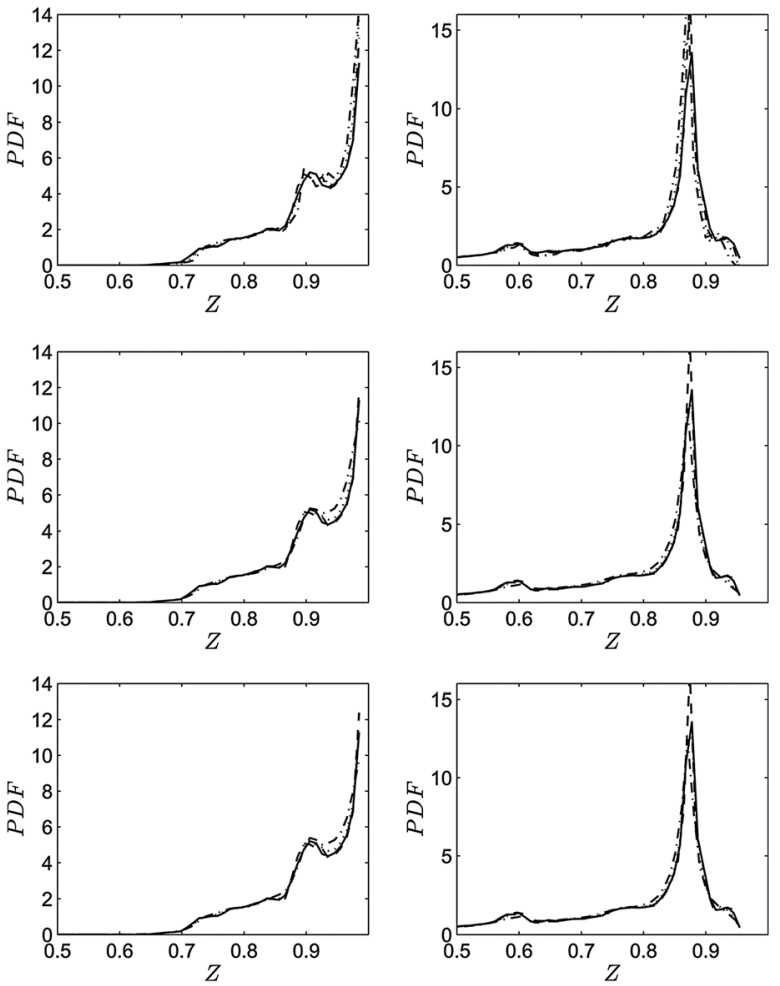

FIG. 5. Filtered PDFs $\left\langle f_{s g s_{c}}\right\rangle_{c}$, from the OH750 database at $t_{t r}^{*}$ over planes of coordinates $x_{2} / \delta_{\omega, 0}=0.44$ (left) and $x_{2} / \delta_{\omega, 0}=5.11$ (right). Exact SGS: solid lines. Assumed PDFs are the Dirac (top), the Gaussian (center), and the Beta (bottom) functions. $\bar{\Delta} / \Delta x_{D N S}=4$, dashed lines; $\bar{\Delta} / \Delta x_{D N S}=8$, dotted lines; $\bar{\Delta} / \Delta x_{D N S}=12$, dot-dashed lines. 

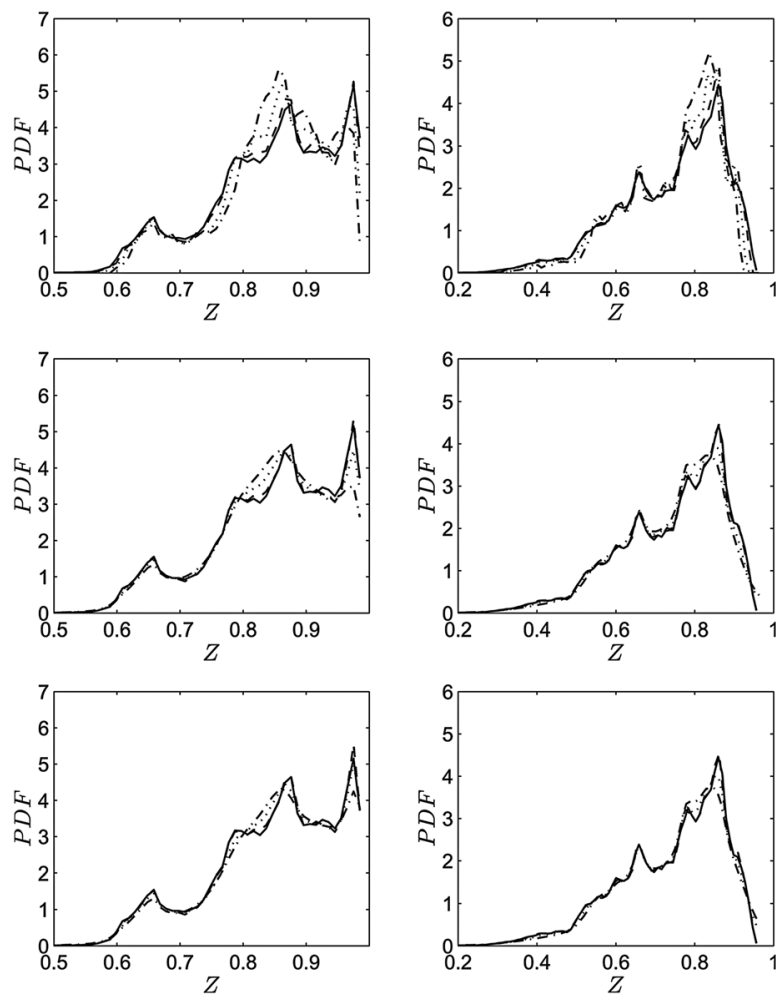

FIG. 6. Filtered PDFs $\left\langle f_{s g s_{c}}\right\rangle_{c}$, from the OHe600 database at $t_{t r}^{*}$ over planes of coordinates $x_{2} / \delta_{\omega, 0}=0.44$ (left) and $x_{2} / \delta_{\omega, 0}=5.11$ (right). Exact SGS: solid lines. Assumed PDFs are the Dirac (top), the Gaussian (center), and the Beta (bottom) functions. $\bar{\Delta} / \Delta x_{D N S}=4$, dashed lines; $\bar{\Delta} / \Delta x_{D N S}=8$, dotted lines; $\bar{\Delta} / \Delta x_{D N S}=12$, dot-dashed lines.

than $\chi_{F}$, particularly at the periphery of the layer. Noteworthy, the linear dependency between $\chi_{T}$ and $\chi_{F}$ in Fig. 3 justifies the use of the scaling law based on the $\widetilde{Z}$ gradient for modeling the SGS scalar variance (see Section VI C 2) since the behavior of $\chi_{T}$ and $\chi_{F}$ is similar.

Considering the rms activity, in concert with the findings from analysis of Eq. (4), the effect of the $D \alpha_{D}$ variation at small scales cannot be underestimated. For HN600 and OHe600 the SGS turbulent fluxes are larger in magnitude than the molecular diffusion flux, meaning that the scalar fluctuations contribute to the mixing at the SGS scales. Among the four SGS diffusivity terms, the second one assumes large values when compared to the other diffusion-like terms or advection term, indicating that it must be retained and modeled in the SGS scalar variance equation; the third and fourth SGS diffusivity terms have smaller magnitudes and their nature is dissipative; finally, the first one compares in magnitude to the resolved diffusion in HN600 and OHe600 but is much larger than the resolved diffusion for $\mathrm{OH750}$. The conclusion is that particular attention should be devoted to SGS diffusivity 1 and SGS diffusivity 2 terms because they have a dominant contribution to the mixing of oxygen with hydrogen at the SGS scales. The Soret contribution, labeled SGS Soret 1, has a diffusion-like behavior and is non negligible only in the HN600 mixing layer. Also, the SGS contributions stemming from filtering of the pressure-gradient terms are negligible in all cases.
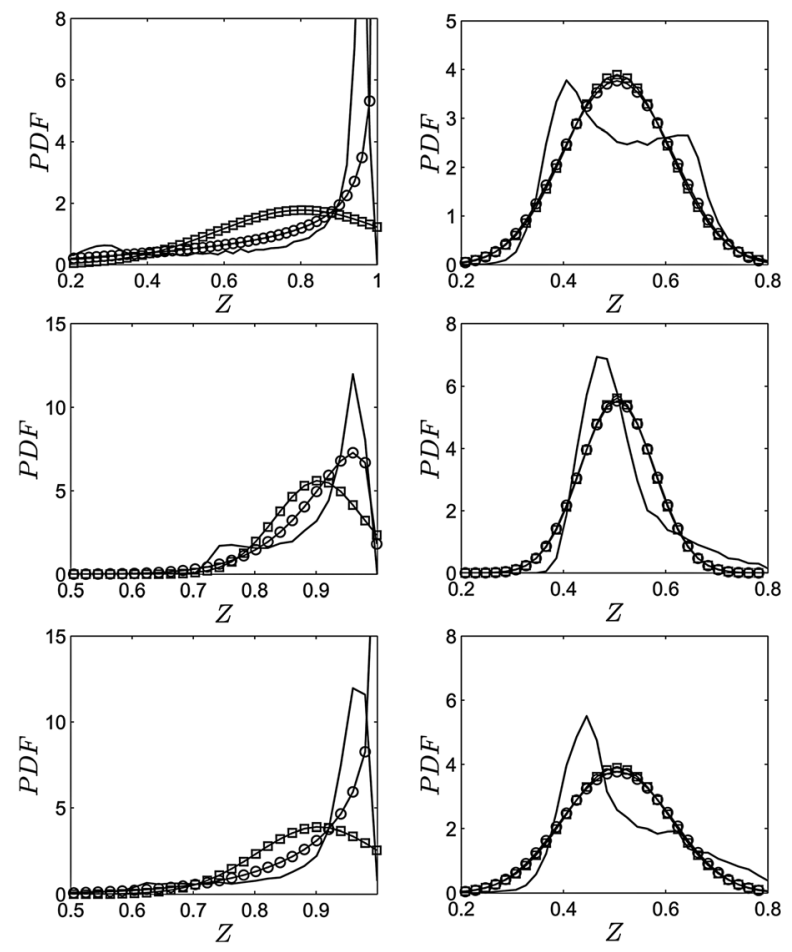

FIG. 7. Conditional expectation of $f_{s g s_{c}}\left(\xi ; \tilde{\xi}, \sigma_{\xi}\right)$ over $\left(\tilde{\xi}, \sigma_{\xi}\right)$ and over the slab at $x_{2} / \delta_{\omega, 0}= \pm 5.3$. Top: HN600, $\tilde{\xi}=0.805 \pm 0.005, \sigma_{\xi}=0.0505$ \pm 0.0005 (left) and $\tilde{\xi}=0.505 \pm 0.005, \sigma_{\xi}=0.0105 \pm 0.0005$ (right). Center: $\mathrm{OH} 750, \quad \tilde{\xi}=0.905 \pm 0.005, \quad \sigma_{\xi}=0.00505 \pm 0.00005 \quad$ (left) and $\tilde{\xi}=0.505 \pm 0.005, \sigma_{\xi}=0.00505 \pm 000005$ (right). Bottom: OHe600, $\tilde{\xi}=0.905 \pm 0.005, \sigma_{\xi}=0.0105 \pm 0.0005$ (left) and $\tilde{\xi}=0.505 \pm 0.005$, $\sigma_{\xi}=0.0105 \pm 0.0005$ (right). The exact result extracted from DNS is shown in solid lines, the Gaussian distribution is represented by squares and the Beta distribution is portrayed by circles. $\bar{\Delta} / \Delta x_{D N S}=8$.

\section{B. Assessment of the presumed-PDF approach for computing filtered non-linear scalar-dependent quantities}

An a priori evaluation of the statistical SGS PDF for the HN600 layer is illustrated in Fig. 4 for the same two $\left(x_{1}, x_{3}\right)$ homogeneous planes of coordinates $x_{2} / \delta_{\omega, 0}=0.44$ and $x_{2} / \delta_{\omega, 0}=5.11$ as in Fig. 3. The DNS-extracted filtered PDF $\left\langle f_{s g s_{c}}\right\rangle_{c}$ is evaluated using the mass-weighted PDF of $Z$ over coarsened-grid planes. The presumed averaged SGS PDFs are computed employing mapped LES moments $\left(\tilde{\xi}, \sigma_{\xi}\right)$ and using Eq. (15). As expected, the figure displays the best prediction when a $\beta$ PDF is used. The difference between onemoment and two-moment distributions is evident both in the central part of the mixing layer $\left(x_{2} / \delta_{\omega, 0}=0.44\right)$ and away from it $\left(x_{2} / \delta_{\omega, 0}=5.11\right)$. The difference between the twomoment distributions, Gaussian, and $\beta$, is also clear, particularly in zones of poor mixing where a Gaussian distribution is not appropriate.

Figure 5 portrays the corresponding $\mathrm{OH} 750$ results over the same $x_{2}$ planes as for the HN600 layer. In the OH750 case, no large difference between PDF models is visible which is conjectured to result from the high hydrogen diffusivity which promotes good mixing. Finally, OHe600 layer results are shown in Figure 6. For OHe mixing, the twomoment PDFs increase the model accuracy compared to the single-moment one, and a $\beta$ PDF gives slightly better results than the Gaussian PDF, as expected. 


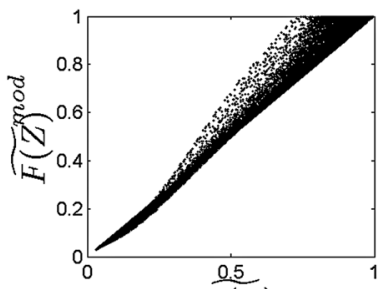

$\frac{55}{F(Z)}$
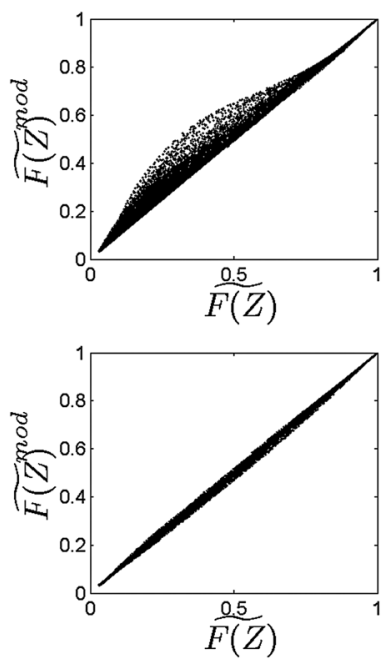

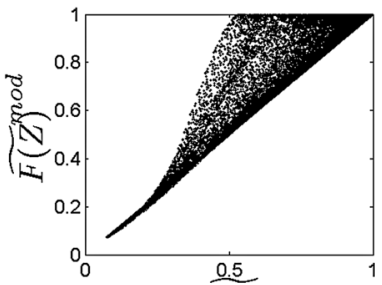

$\overline{F(Z)}$
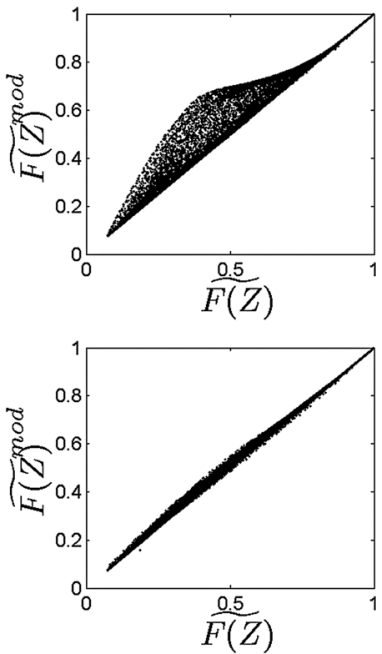

FIG. 8. Scatter plot of the modeled function $\widetilde{F(Z)}$ mod versus the exact quantity $\widehat{F(Z)}$ computed over planes $x_{2} / \delta_{\omega, 0}=0.44$ (left) and $x_{2} / \delta_{\omega, 0}=5.11$ (right). The exact quantity is the filtered HN600 DNS at $t_{t r}^{*}$, and the models are the Dirac PDF (top), Gaussian PDF (center), and $\beta$ PDF (bottom). $\bar{\Delta} / \Delta x_{D N S}=8$.

Results from the optimal estimators are displayed in Fig. 7. Because this methodology is based on conditioning over a couple of moments, the Dirac presumed PDF is not considered. In order to increase the sample size, the conditional expectations are computed over a slab of the mixing layer of coordinates $x_{2} / \delta_{\omega, 0}= \pm 5.3$. As expected, in regions of well-mixed species, the $\beta$ PDF and the Gaussian PDF give similar predictions while the $\beta \mathrm{PDF}$ is more appropriate for unmixed situations.

Thus, we have shown that two methodologies are in agreement regarding the appropriateness of the presumed $\beta$ PDF shape. However, it is known that for atmospheric conditions ${ }^{17}$ good agreement with a template for averaged distributions (as given by both methodologies) does not necessarily imply similar pointwise agreement, so it is important to test the capabilities of the three distributions of Eqs. (16), (18), and (19) to provide local agreement (as needed in reactive flows) in computations where they would be used to reproduce filtered non-linear terms (e.g., reaction rates). For this purpose, we select a simple non-linear $Z$ function

$$
F(\xi)=\exp \left\{-2\left[\operatorname{erf}^{-1}(2 \xi-1)\right]^{2}\right\}
$$

representing the $\chi$ functional form in a one-dimensional unsteady laminar subcritical mixing layer. ${ }^{47}$ Then, the filtered value of $F(\xi)$ may be estimated by integration over the scalar space using Eq. (13) as
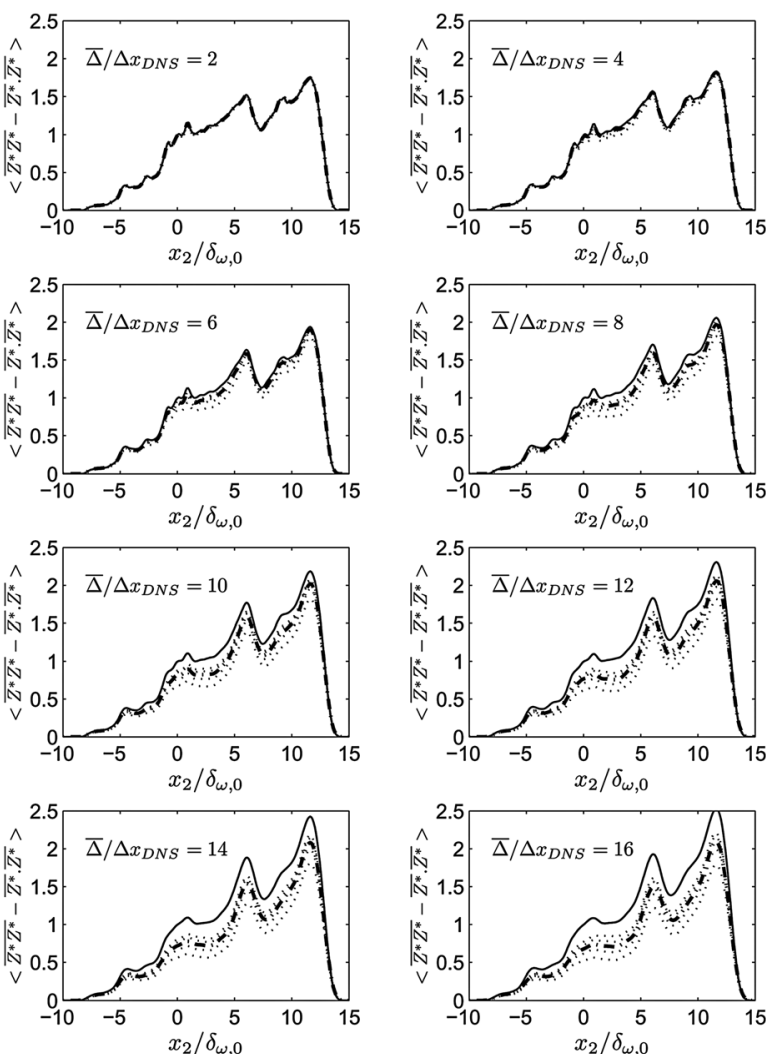

FIG. 9. Predictions of the SGS scalar variance using the deconvoluted field $\mathrm{Z}^{*}$. Several orders of approximation are shown for different filter widths at $t_{t r}^{*}$ for the HN600 mixing layer. Exact SGS scalar variance: solid line. Dotted lines are the first to fifth order approximations, the third order being distinguished by a dash-dotted line. Variances are non-dimensionalized by the exact value at the center of the mixing layer. Computation performed using Eq. (21).

$$
\widetilde{F(\mathbf{x})}=\int_{0}^{1} F(\xi) f_{s g s_{c}}(\xi ; \mathbf{x}) d \xi .
$$

Figure 8 illustrates scatter plots of the modeled quantity $\widetilde{F(Z)^{\text {mod }}}$ (superscript mod labels a modeled quantity) versus the exact quantity $\widetilde{F(Z)}$ extracted from the filtered DNS database HN600, over two planes, in the central part and at the periphery of the layer. The modeled functions are computed by replacing $f_{\text {sgs }}(\xi ; \mathbf{x})$ in Eq. (52) with the presumed SGS PDF (Dirac, Gaussian, or $\beta$ ) constructed from the exact moments extracted from the filtered DNS database at $t_{t r}^{*}$. The local results are in accord with the assessment of the statistical SGS PDFs presented in Figs. 4 and 7. A drastic improvement in predictions is obtained by using the $\beta$-PDF in the mixing of heptane and nitrogen. An improvement is also obtained for the oxygen/helium system (not shown) while in the oxygen/hydrogen mixing layer the differences are minor (not shown).

As mentioned in Sec. III, a clipped Gaussian was also considered. For the filtered PDF, results using the clipped Gaussian exhibited small differences from those obtained with the truncated Gaussian for the HN600 mixing layer (not shown), and imperceptible differences for the OH750 and OHe600 mixing layers (not shown). The clipped Gaussian also provided slightly more accurate results when used to model the non-linear Z-function (not shown). 

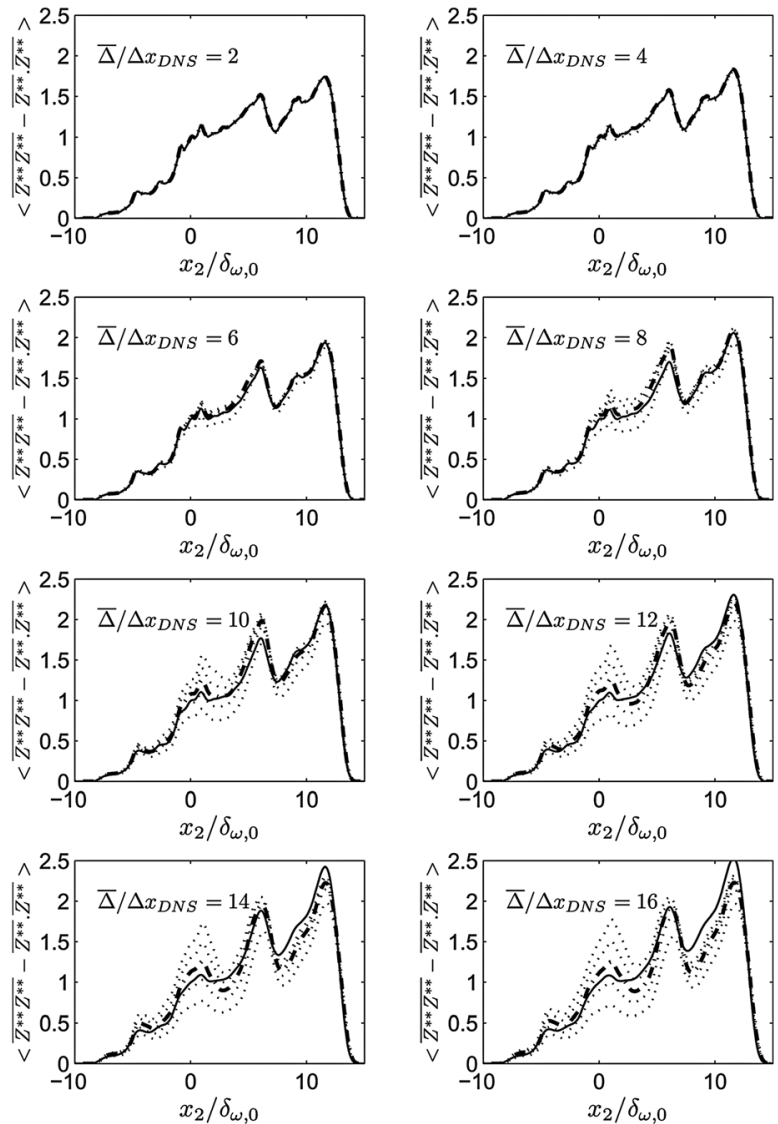

FIG. 10. Predictions of the SGS scalar variance using the deconvoluted field $\mathrm{Z}^{* *}$. Several orders of approximation are shown for different filter widths at $t_{t r}^{*}$ for the HN600 mixing layer. Exact SGS scalar variance: solid line. Dotted lines are the first to fifth order approximations, the third order being distinguished by a dash-dotted line. Variances are non-dimensionalized by the exact value at the center of the mixing layer. Computation performed using $\mathrm{Z}^{* *}$ extracted from DNS.

Whereas the Z-function of Eq. (51) is here used only to test the ability of the models to reproduce nonlinearities, the function is not necessarily expected to represent the real $\chi$ for three-dimensional supercritical flows. This topic is addressed in Appendix A.

\section{Evaluation of direct models for the SGS scalar variance}

Having shown in Sec. VI A that under supercritical $p$ conditions modeling of new terms in the $\sigma_{Z}$ equation is necessary (but uncertain), and shown in Sec. VI B that wellknown assumed $\beta$ PDFs may be used in modeling nonlinear scalar-dependent functions, the next step is to assess models for computing $\sigma_{Z}$ from the filtered DNS solution to enable the construction of the presumed SGS PDFs. Results from two such models are presented below.

\section{Evaluation of the approximate deconvolution model}

All presented results were computed on the DNS (rather than LES) grid. In all figures, the information is shown for several $\bar{\Delta} / \Delta x_{D N S}$ values and for each $\bar{\Delta} / \Delta x_{D N S}$ value for five orders of reconstruction.

Since Eq. (24) is at the core of the compressible ADM procedure, we first inquired about the convergence of $Z^{* *}$ to
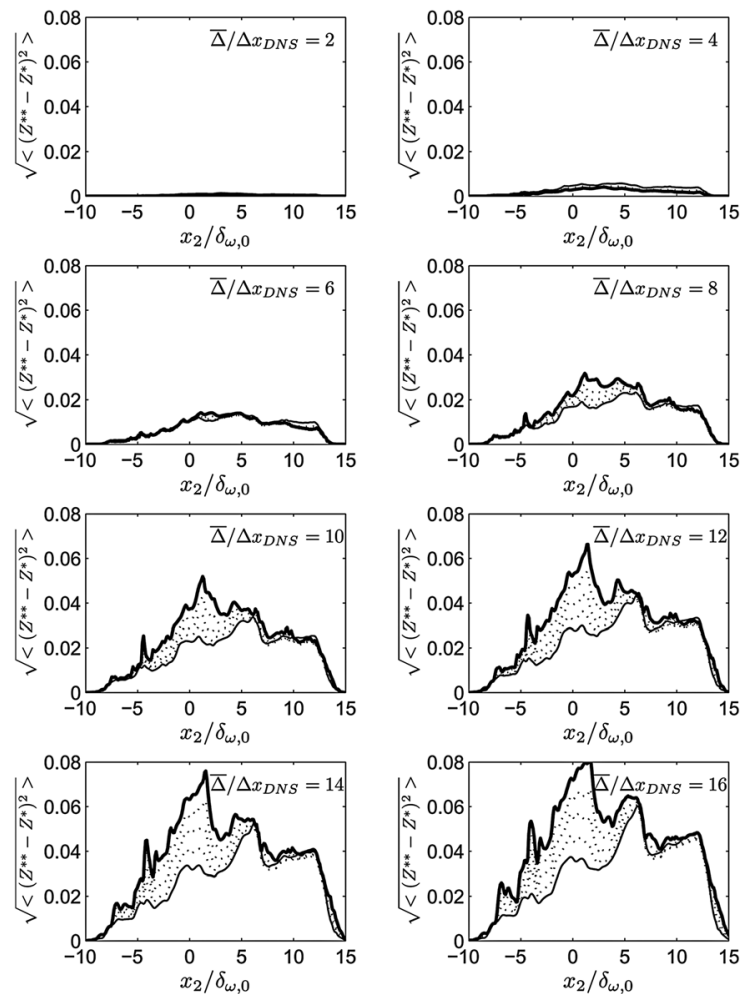

FIG. 11. Planar rms activity of the field $\left(Z^{* *}-Z^{*}\right)$ for several orders of ADM approximation and different filter widths at $t_{t r}^{*}$ for the HN600 mixing layer. Thin solid line: first order of approximation; thick solid line: fifth order of approximation. Dotted lines are the second to fourth order approximations.
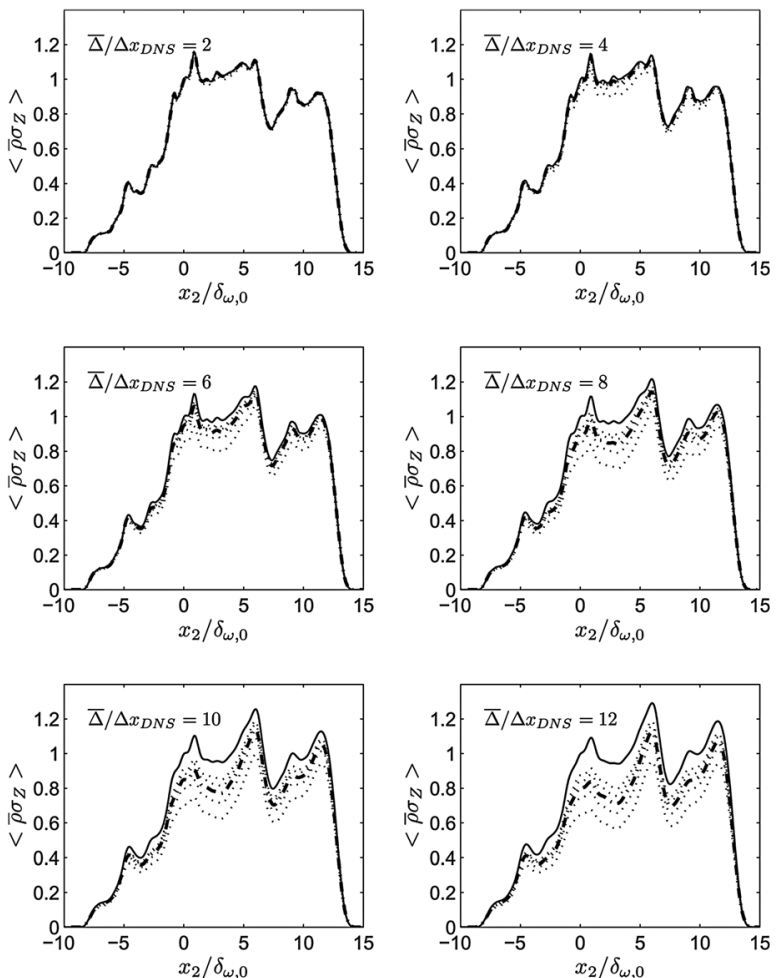

FIG. 12. Predictions of the Favre SGS scalar variance using ADM applied to the primitive quantity. Several orders of approximation are shown for different filter widths at $t_{t r}^{*}$ for the HN600 mixing layer. Exact SGS scalar variance: solid line. Dotted lines are the first to fifth order approximations, the third order being distinguished by a dash-dotted line. Variances are nondimensionalized by the exact value at the center of the mixing layer. 

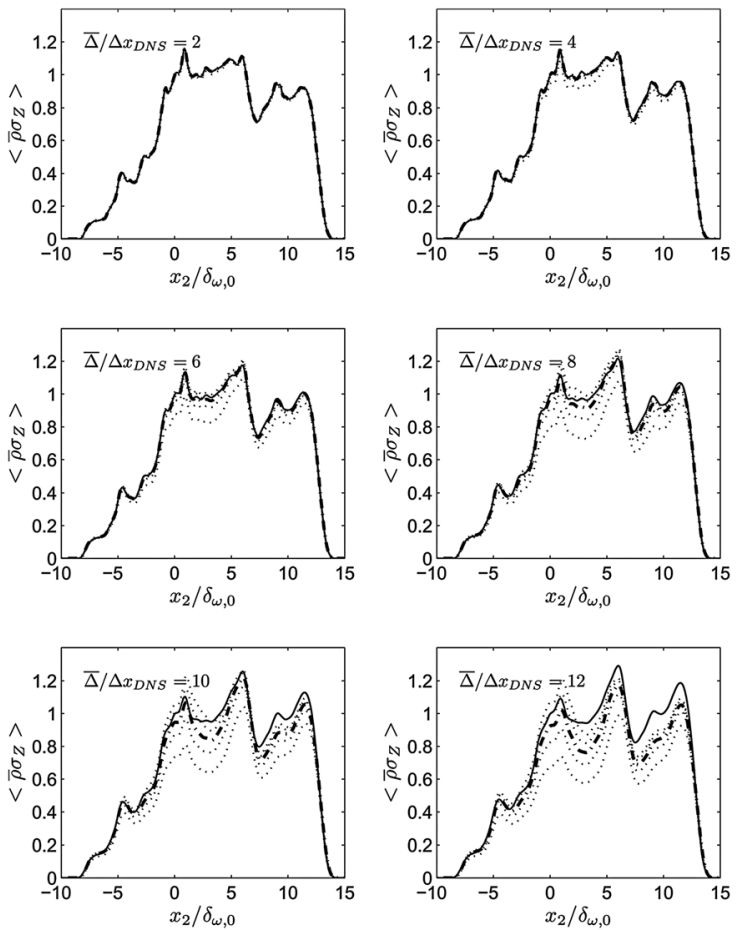

FIG. 13. Predictions of the Favre SGS scalar variance using ADM applied to conservative quantities. Several orders of approximation are shown for different filter widths at $t_{t r}^{*}$ for the HN600 mixing layer. Exact SGS scalar variance: solid line. Dotted lines are the first to fifth order approximations, the third order being distinguished by a dash-dotted line. Variances are nondimensionalized by the exact value at the center of the mixing layer.
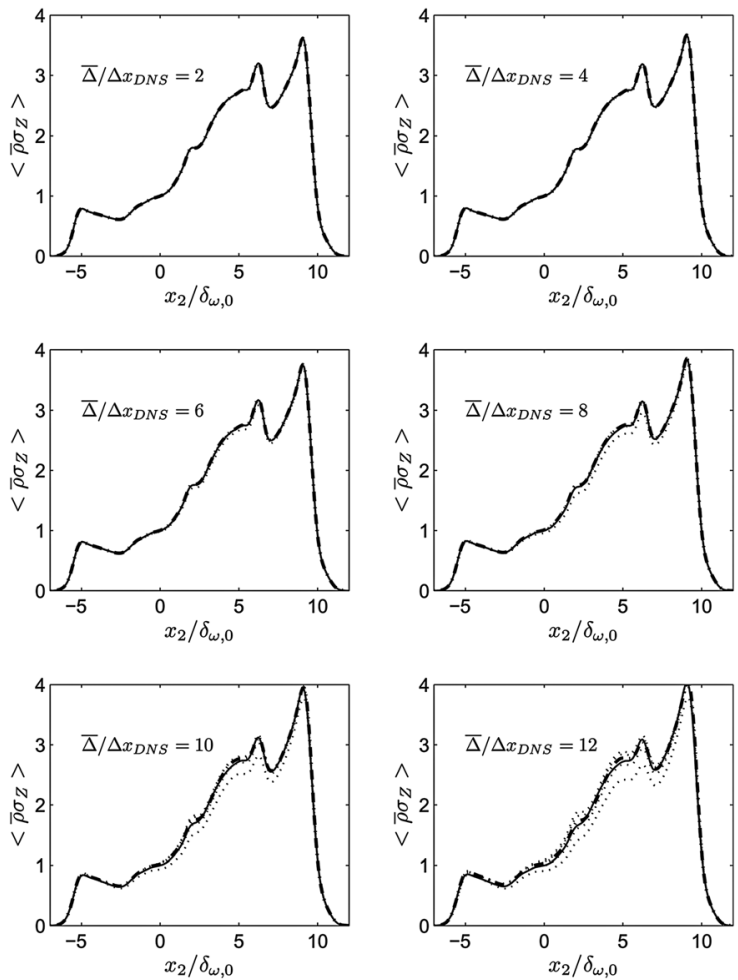

FIG. 14. Predictions of the Favre SGS scalar variance using ADM applied to conservative quantities. Several orders of approximation are shown for different filter widths at $t_{t r}^{*}$ for the OH750 mixing layer. Exact SGS scalar variance: solid line. Dotted lines are the first to fifth order approximations, the third order being distinguished by a dash-dotted line. Variances are nondimensionalized by the exact value at the center of the mixing layer.
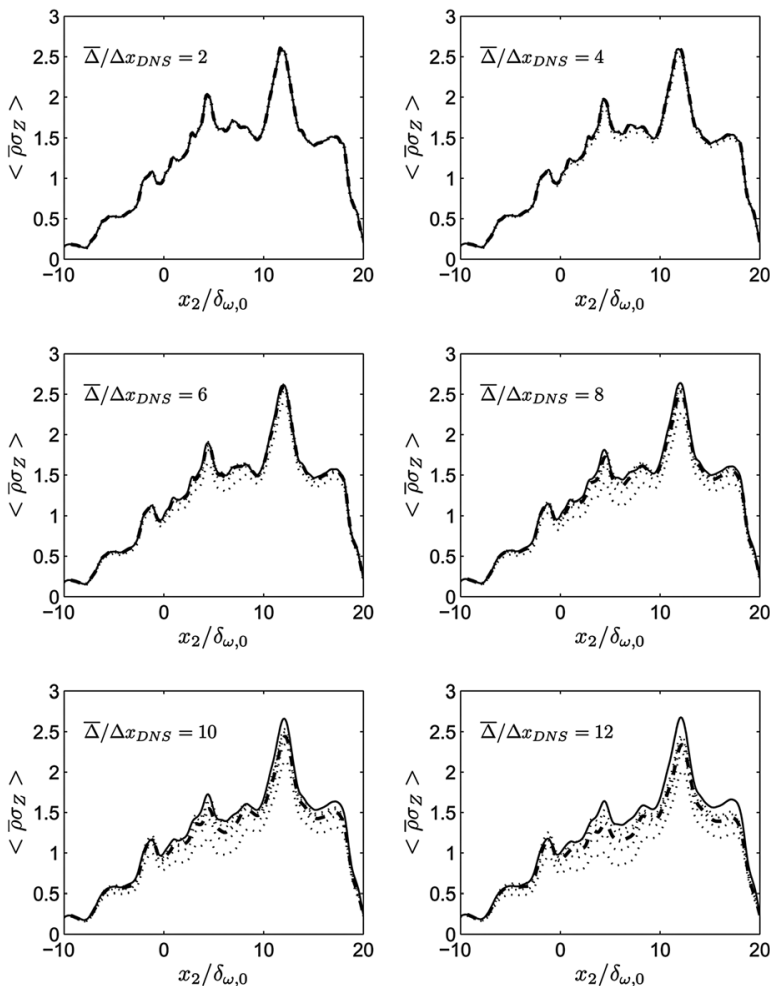

FIG. 15. Predictions of the Favre SGS scalar variance using ADM applied to conservative quantities. Several orders of approximation are shown for different filter widths at $t_{t r}^{*}$ for the OHe600 mixing layer. Exact SGS scalar variance: solid line. Dotted lines are the first to fifth order approximations, the third order being distinguished by a dash-dotted line. Variances are nondimensionalized by the exact value at the center of the mixing layer.
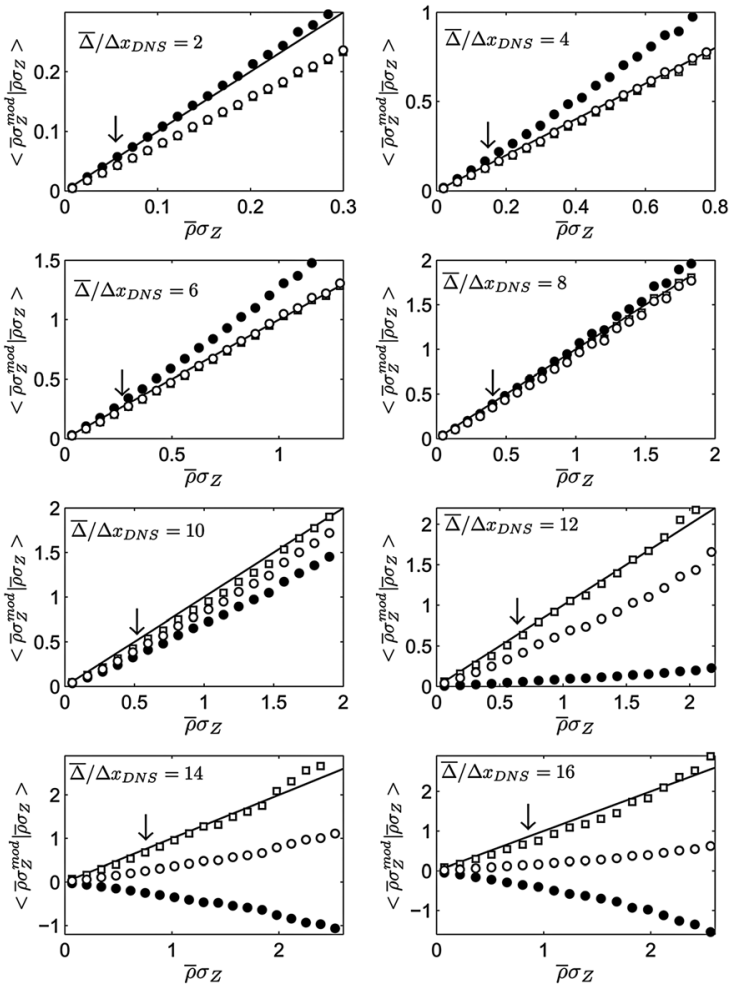

FIG. 16. Planar averages of modeled SGS scalar variances conditioned on the exact ones, evaluated using the filtered HN600 DNS at $t_{t r}^{*}$, over the plane $x_{2} / \delta_{\omega, 0}=0.44$. Coefficients are computed using the new model (Eq. (48)) (squares), and the classical model (Eq. (47)) with either $\widehat{\bar{\Delta}}^{2}=\widehat{\Delta}^{2}+\bar{\Delta}^{2}$ (empty circles) or $\widehat{\bar{\Delta}}=\widehat{\Delta}$ (filled circles). The arrow indicates the mean quantity $\left\langle\bar{\rho} \sigma_{Z}\right\rangle$. 

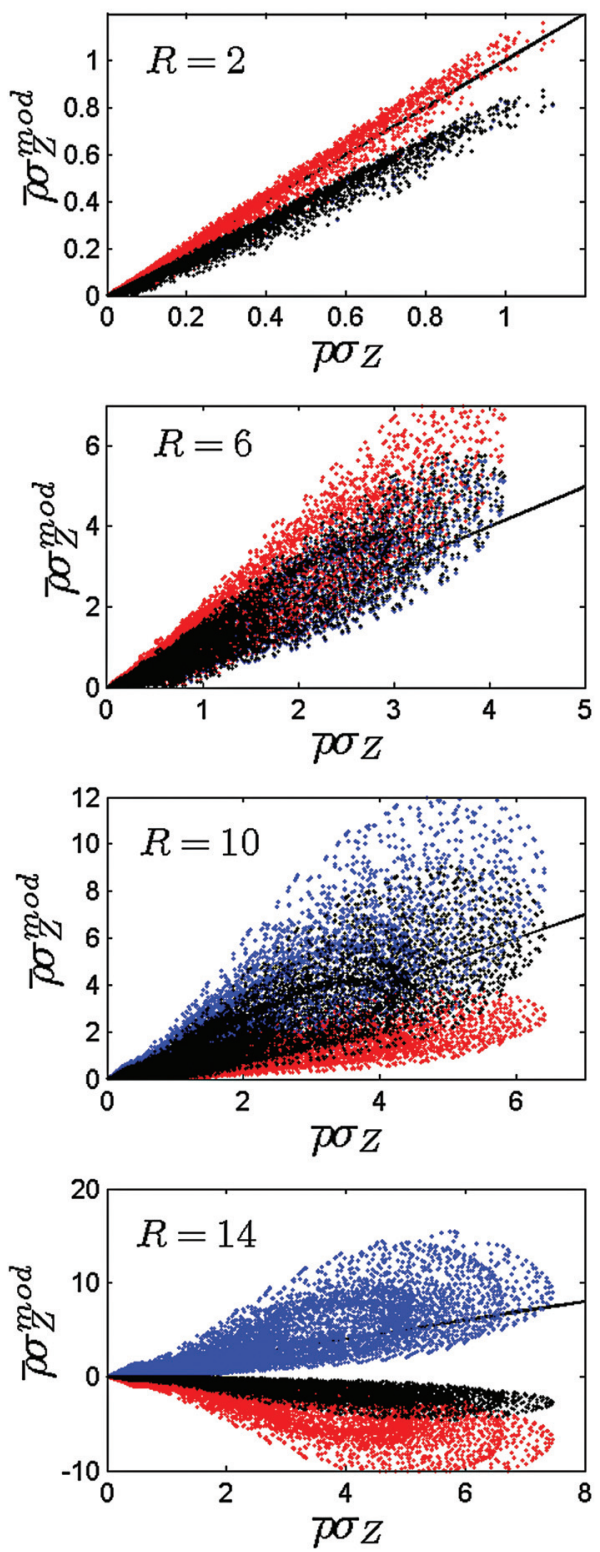
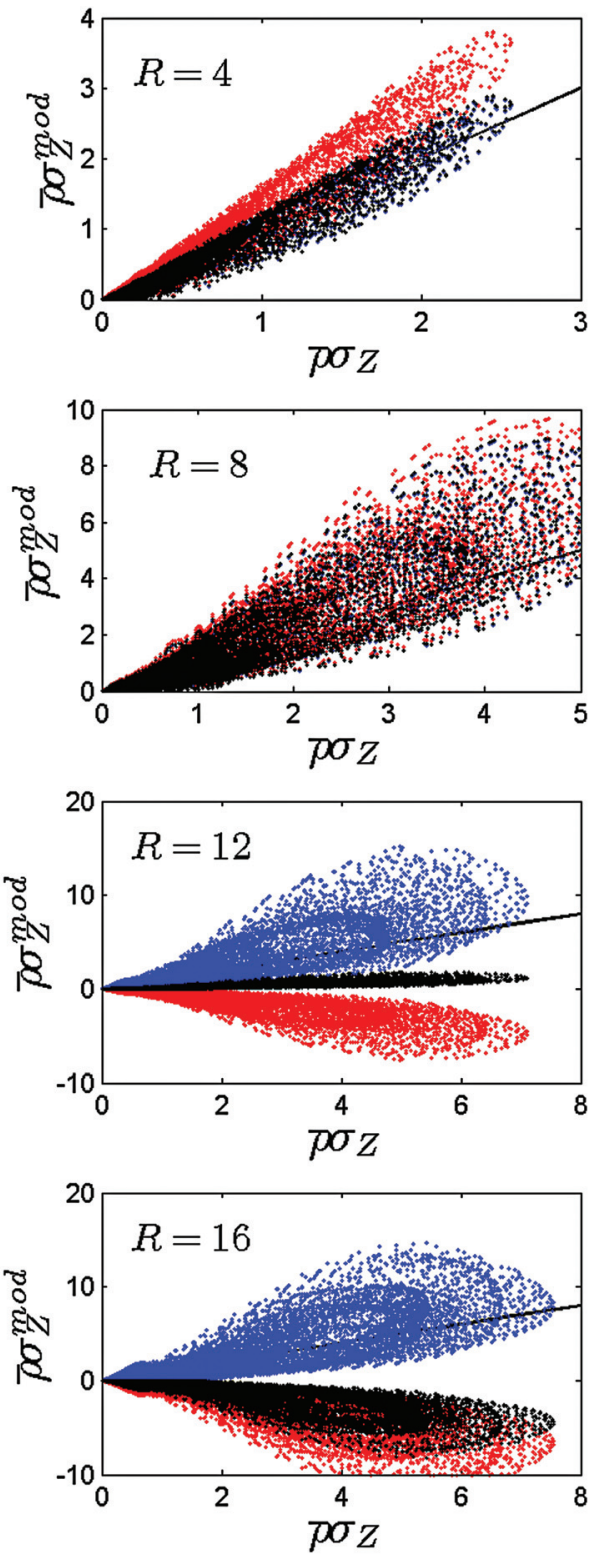

FIG. 17. (Color) Scatter plot of modeled against exact SGS scalar variance evaluated using the filtered HN600 DNS at $t_{t r}^{*}$, over the plane $x_{2} / \delta_{\omega, 0}=5.11$. Coefficients are computed using the new model (Eq. (48)) (blue symbols), and the classical model (Eq. (47)) with either $\bar{\Delta}^{2}=\widehat{\Delta}^{2}+\bar{\Delta}^{2}$ (black symbols) or $\bar{\Delta}=\widehat{\Delta}$ (red symbols). $R \equiv \bar{\Delta} /$ $\triangle x_{D N S}$.
$Z$ according to the approximation of Eq. (25). To explore this issue, we first computed the local second-order moment of $Z^{*}$ and compared it to the exact moment extracted from the filtered DNS database of the HN600 mixing layer at $t_{t r}^{*}$; the results are illustrated in Fig. 9. Then, we calculated the local second-order moment of $Z^{* *}$ and compared it to the exact moment extracted from the filtered DNS database of the HN600 mixing layer at $t_{t r}^{*}$; the results are illustrated in Fig. 10. Whereas the moment of $Z^{*}$ converges to that of $Z$ as $N$ increases, the observations are that the moment of the deconvoluted approximate field $Z^{* *}$ does not converge to that of $Z$ and that in some zones this second-order moment is overestimated. This overestimate occurs for a filter width as small as $6 \Delta x_{D N S}$, and the situation deteriorates with increasing values of $\bar{\Delta} / \Delta x_{D N S}$. The rms activity of the difference between $Z^{* *}$ and $Z^{*}$ is presented in Fig. 11. Clearly, this difference increases with the filter width, and it also globally increases with $N$. In order to inquire about the impact of the
$Z^{* *} \simeq Z^{*}$ approximation on the SGS-scalar-variance predictions for compressible flows, the ADM procedure is tested using the Favre SGS scalar variance.

The results of Fig. 12 portray the ADM procedure applied to the primitive variable $\bar{Z}$ as in Eq. (21), and the SGS scalar variance is computed using the exact local density and the approximate field $Z^{*}$ as $\sigma_{Z}=$ $\left(\overline{\rho Z^{*} Z^{*}} / \bar{\rho}\right)-\left(\overline{\rho Z^{*}} / \bar{\rho}\right)\left(\overline{\rho Z^{*}} / \bar{\rho}\right)$. The results of Fig. 13 differ from those of Fig. 12 in that the ADM procedure is applied to both conservative variables $\bar{\rho}$ and $\overline{\rho Z}$, as one would do in a practical case, and the reconstructed density $\rho^{*}$ with the approximate field $Z^{* *}$ are used to compute the SGS scalar variance according to Eq. (26). Comparing Figs. 12 and 13 at same $\bar{\Delta} / \Delta x_{D N S}$ value, it is clear that using the ADM on the conservative variables improves the model predictions. For example, for $\bar{\Delta} / \Delta x_{D N S}=8$, a third-order approximation is sufficient for reproducing $\left\langle\bar{\rho} \sigma_{Z}\right\rangle$ at the center of the mixing layer, while by using the primitive quantities only $90 \%$ of its 

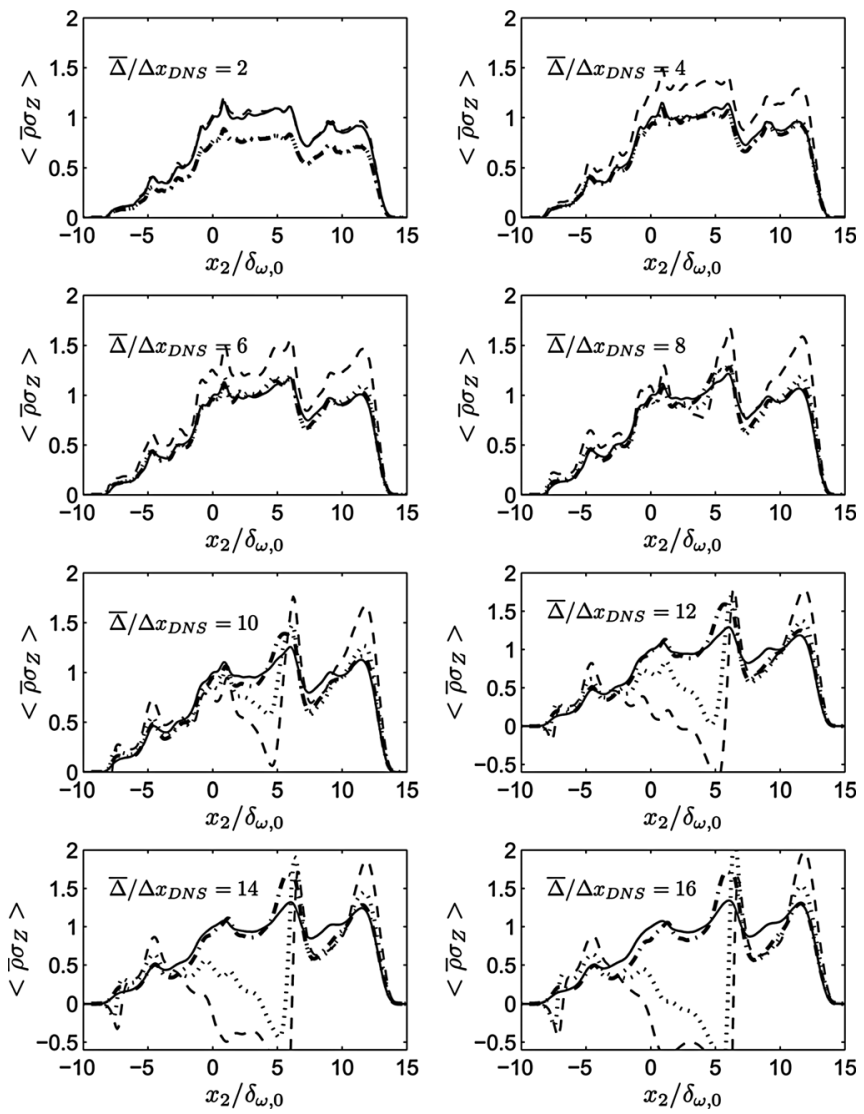

FIG. 18. Profiles of modeled and exact SGS scalar variances, evaluated using the filtered HN600 DNS at $t_{t r}^{*}$. Exact values: solid line. New model: dash-dotted line. Classical model using $\widehat{\Delta}^{2}=\widehat{\Delta}^{2}+\bar{\Delta}^{2}$ : dotted line. Classical model using $\widehat{\Delta}=\widehat{\Delta}$ : dashed line. Variances are non-dimensionalized by the exact value at the center of the mixing layer.

value is recovered. The difference between the ADM based on primitive variables and that based on conservative variables becomes enlarged as the filter width increases. For small filter widths $\left(\bar{\Delta} / \Delta x_{D N S}=2,4,6\right)$, a second-order reconstruction (3 terms) gives very good agreement compared to the exact value. Similarly to the observation of Pantano and Sar$\mathrm{kar}^{16}$ for incompressible flows, the ADM accuracy depends at least on the level of turbulence, i.e., the Reynolds number, and on the filter width; here, there is the additional complication of variable density which introduces Eq. (24). Figures 14 and 15 show corresponding results for $\mathrm{OH} 750$ and OHe600, respectively, when the ADM is performed using the conservative variables.

Despite the better performance of the ADM conservative-variable based model compared to the primitive-variable based one for the third-order approximation, the convergence issue discussed above is still an item of concern when using the approximation Eq. (25). One conclusion from the presented assessment is that users of the ADM should be cautious when employing this methodology for conservative variables in the context of compressible flows, and results should be carefully verified. Here, because the overestimate of the SGS second-order moment of $Z^{* * *}$ is combined with an underestimate of $\rho^{*}$, the global result is a satisfactory prediction of the SGS (Favre) scalar variance. However, ADM
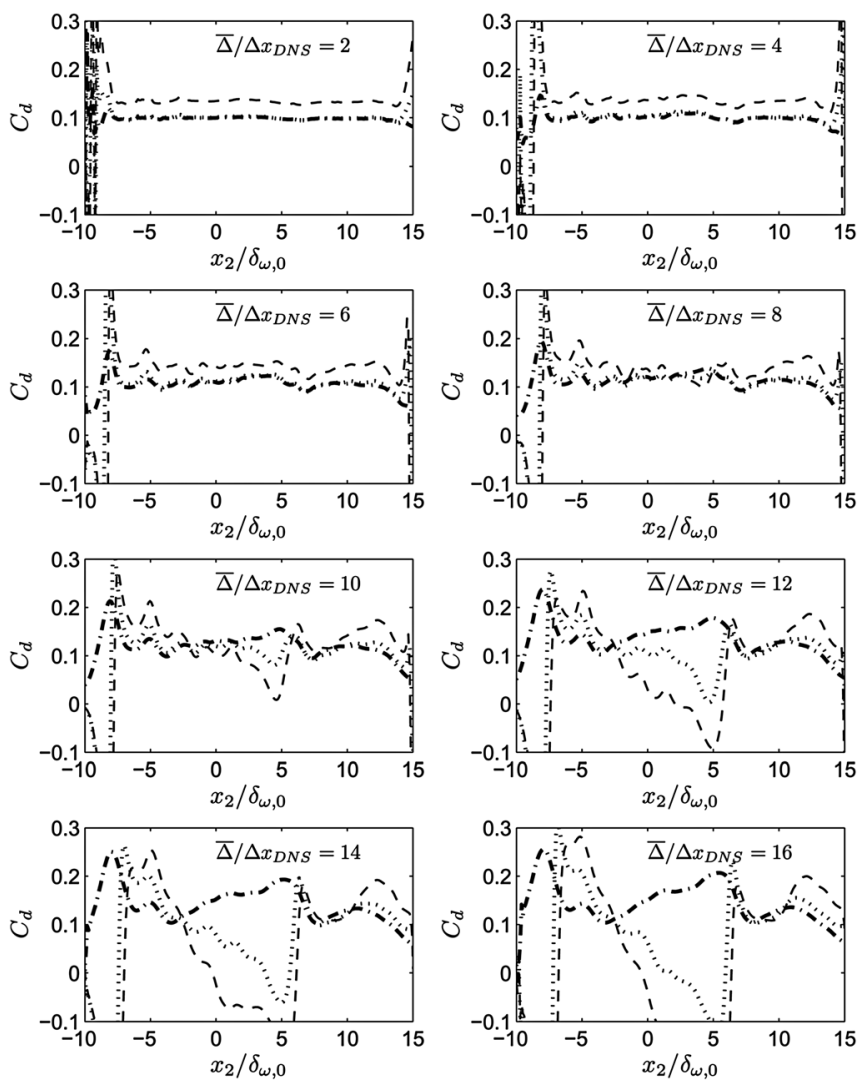

FIG. 19. Model coefficients $C_{d}$ evaluated using the filtered HN600 DNS at $t_{t r}^{*}$. New model: dash-dotted line. Classical model using with $\bar{\Delta}^{2}=\widehat{\Delta}^{2}+\bar{\Delta}^{2}$ : dotted line. Classical model using $\widehat{\bar{\Delta}}=\widehat{\Delta}$ : dashed line.

should only be considered here as an approximation rather than an asymptotically convergent expansion.

\section{Evaluation of the dynamic gradient-based model}

The ratio of the test-filter width to grid-filter width used in this study is $\hat{\Delta} / \bar{\Delta}=2$.

Figure 16 depicts planar averages of the modeled SGS scalar variance conditioned on the exact one extracted from the filtered HN600 DNS database. The plots represent averages at $t_{t r}^{*}$ over a plane close to the center of the mixing layer $\left(x_{2} / \delta_{\omega, 0}=0.44\right)$ as a function of $\bar{\rho} \sigma_{Z}$, for several filter widths; the vertical arrow represents $\left\langle\bar{\rho} \sigma_{Z}\right\rangle$ and provides an indication of the model fidelity at that particular value. For $\bar{\Delta} / \Delta x_{D N S}=2$, filtering is clearly performed in the dissipation range, whereas, as an example, for $\bar{\Delta} / \Delta x_{D N S}=14,16$ testfiltering is performed close to the production range, and thus neither of these values are in concert with SGS modeling assumptions, but they are here presented for illustrative purposes. At $\bar{\Delta} / \Delta x_{D N S}=2$, the model based on the Leonard term expansion agrees with the classical model used in conjunction with the correct filter width, but neither one of the models reproduces the exact value, which is better rendered by the classical model utilized in conjunction with an incorrect filter width; this result should serve as a warning that if SGS modeling is tested in the incorrect wavenumber range, results from this test are not necessarily reliable. Over the $\bar{\Delta} / \Delta x_{D N S}=4$ to 8 range, the new model and the classical model using the correct filter width agree and additionally 

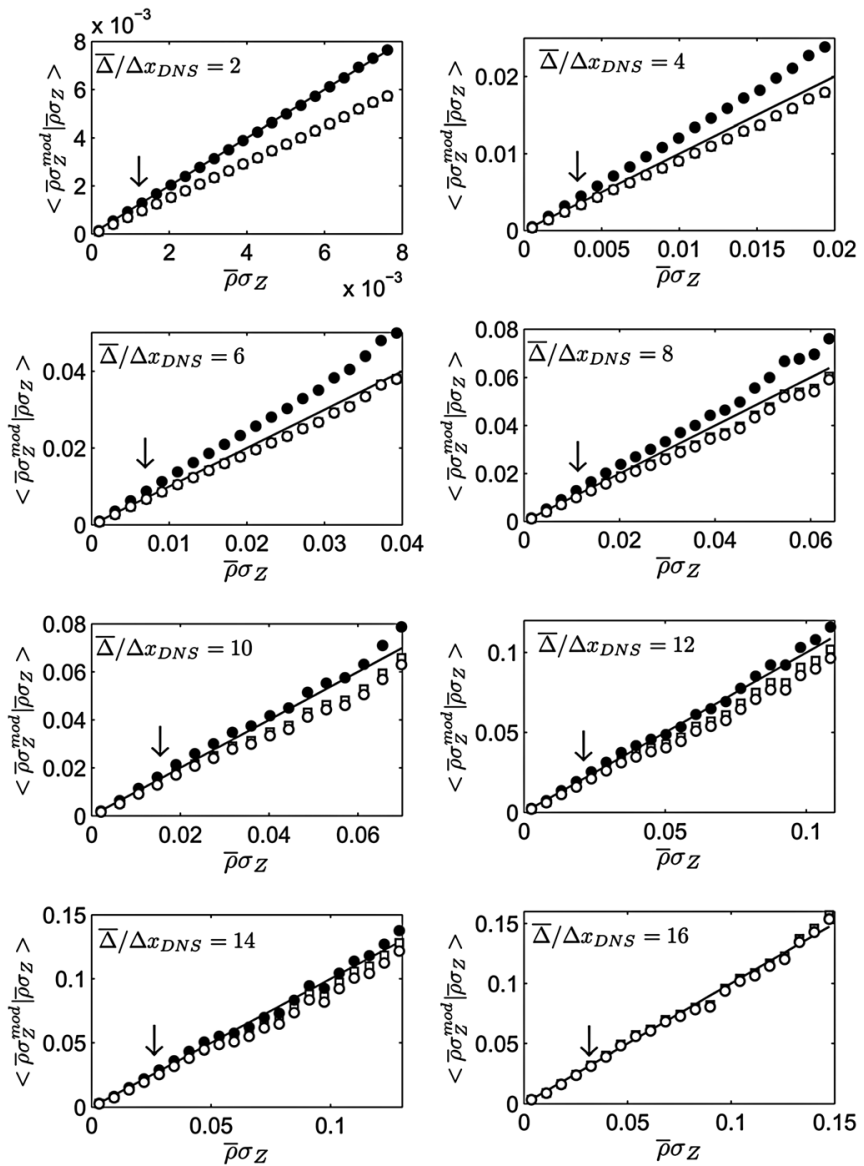

FIG. 20. Planar averages of modeled SGS scalar variances conditioned on the exact ones evaluated using the filtered OH750 DNS at $t_{t r}^{*}$ over the plane $x_{2} / \delta_{\omega, 0}=0.44$. Coefficients are computed using the new model (Eq. (48)) (squares) and the classical model (Eq. (47)) with either $\bar{\Delta}^{2}=\widehat{\Delta}^{2}+\bar{\Delta}^{2}$ (empty circles) or $\widehat{\Delta}=\widehat{\Delta}$ (filled circles). The arrow indicates the mean quantity $\left\langle\bar{\rho} \sigma_{Z}\right\rangle$.

reproduce the DNS-extracted value, whereas the classical model utilized with the incorrect filter width overpredicts it; however, the $\left\langle\bar{\rho} \sigma_{Z}\right\rangle$ value is equally well predicted by all models. For $\bar{\Delta} / \Delta x_{D N S}=10,12$, the fidelity of the new model to predict the exact $\left\langle\bar{\rho} \sigma_{Z}\right\rangle$ is maintained, but that of the classical model with the correct filter deteriorates by underpredicting the template, and for $\bar{\Delta} / \Delta x_{D N S}=12$ severe underpredictions are obtained with the classical model in conjunction with the incorrect filter width. Most important, the classical model used with either filter widths produces incorrect values even for $\left\langle\bar{\rho} \sigma_{Z}\right\rangle$, whereas the new model maintains high fidelity for this quantity. The robustness of the new model is highlighted by the $\bar{\Delta} / \Delta x_{D N S}=14,16$ results where its predictions are still excellent, whereas those with the classical model used with the correct filter width display severe deteriorations and those of the classical model using the incorrect filter width are totally compromised by producing negative values of the SGS scalar variance. Similar to the $\bar{\Delta} / \Delta x_{D N S}=10,12$ situation, $\left\langle\bar{\rho} \sigma_{Z}\right\rangle$ is correctly only predicted by the new model, and negative values of the SGS scalar variance are exhibited by the classical model in conjunction with the incorrect filter width. To show that these comparisons are not $x_{2}$-plane dependent, scatter plots at $t_{t r}^{*}$ of modeled SGS scalar variances against actual values for
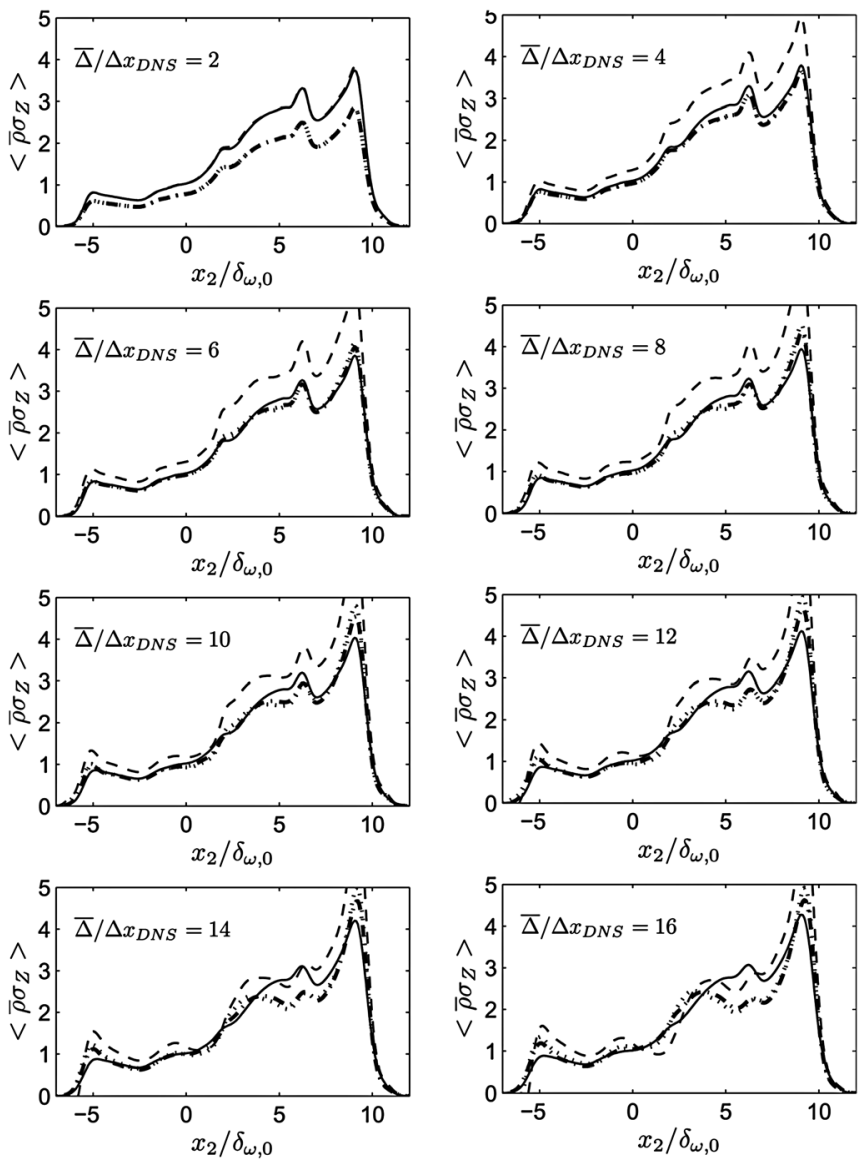

FIG. 21. Profiles of modeled and exact SGS scalar variances evaluated using the filtered OH750 DNS at $t_{t r}^{*}$. Exact values: solid line. New model: dash-dotted line. Classical model using $\widehat{\Delta}^{2}=\widehat{\Delta}^{2}+\bar{\Delta}^{2}$ : dotted line. Classical model using $\bar{\Delta}=\widehat{\Delta}$ : dashed line. Variances are non-dimensionalized by the exact value at the center of the mixing layer.

several filter widths, over a plane close to the periphery of the mixing layer $\left(x_{2} / \delta_{\omega, 0}=5.11\right)$ are illustrated in Fig. 17. Only for $\bar{\Delta} / \Delta x_{D N S}=8$ do the scatter plots overlap for all three models, and predicted negative variances by the classical model using the incorrect filter width appear for a filter ratio of $\bar{\Delta} / \Delta x_{D N S}=12$ which is smaller than the 14 and 16 where we found negative values in the $x_{2} / \delta_{\omega, 0}=0.44$ plane.

The Figs. 16 and 17 results were for two selected $x_{2}$ planes. To further evaluate the potential of the various models we illustrate in Fig. 18 comparisons of the modeled $\left\langle\bar{\rho} \sigma_{Z}\right\rangle$ with the exact one for the same $\bar{\Delta} / \Delta x_{D N S}$ values and for the entire $x_{2}$ significant range. The advantage of the new model over the classical model using the correct filter width is evident for as small value as $\bar{\Delta} / \Delta x_{D N S}=8$, and comparing with the classical model using the incorrect filter width for as small value as $\bar{\Delta} / \Delta x_{D N S}=4$. The high fidelity of the new model persists at large $\bar{\Delta} / \Delta x_{D N S}$ whereas it substantially deteriorates for the other two models with increasing $\bar{\Delta} / \Delta x_{D N S}$ values. The model coefficients computed with the three models are depicted in Fig. 19. The indications are that over all $x_{2}$-planes of the mixing layer, the use of the Leonard term expansion for the dynamic model yields model coefficient values which span a smaller range at fixed $\bar{\Delta} / \Delta x_{D N S}$ value than those of the classical model, thereby showing greater potential in maintaining stability of a LES computation. 

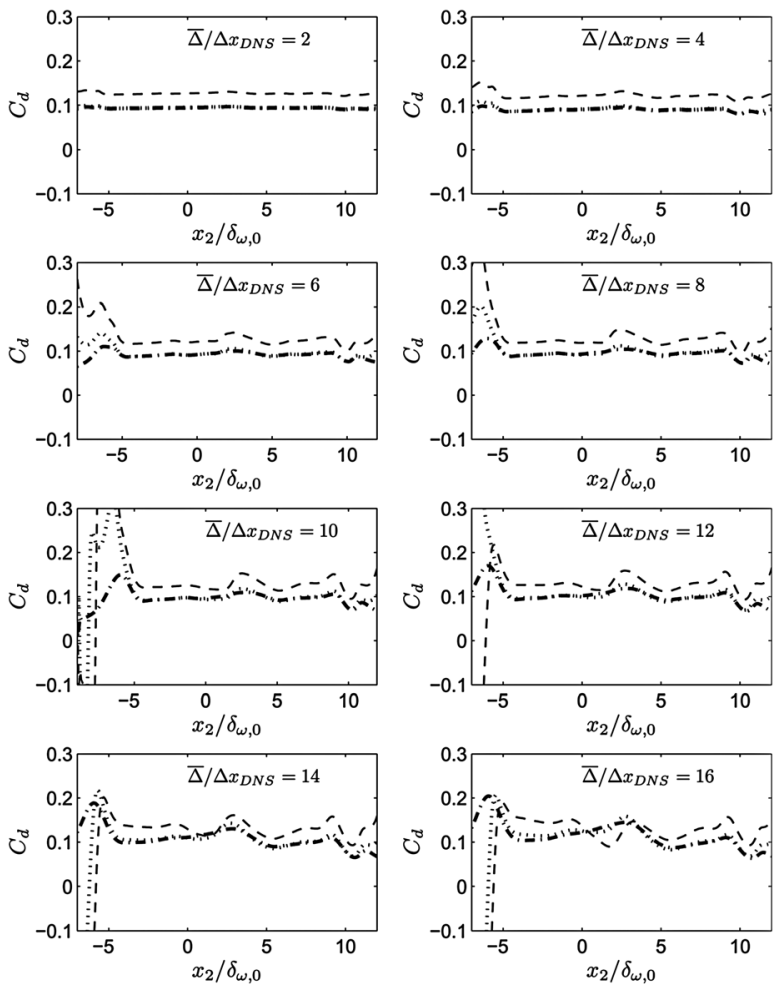

FIG. 22. Model coefficients $C_{d}$ evaluated using the filtered OH750 DNS at $t_{t r}^{*}$. New model: dash-dotted line. Classical model using $\widehat{\Delta}^{2}=\widehat{\Delta}^{2}+\bar{\Delta}^{2}$ : dotted line. Classical model using $\widehat{\bar{\Delta}}=\widehat{\Delta}$ : dashed line.
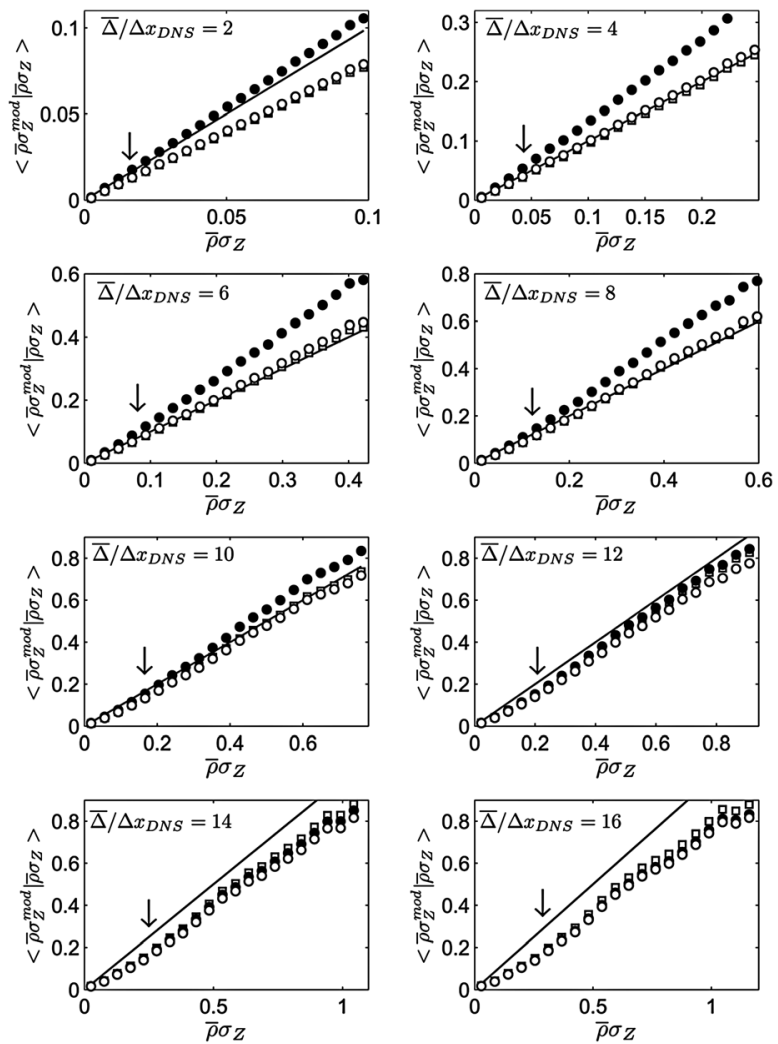

FIG. 23. Planar averages of modeled SGS scalar variances conditional to exact ones evaluated using the OHe600 DNS at $t_{t r}^{*}$ over the plane $x_{2} / \delta_{\omega, 0}=0.44$. Coefficients are computed using the new model (Eq. (48)) (squares) and the classical model (Eq. (47)) with either $\bar{\Delta}^{2}=\widehat{\Delta}^{2}+\bar{\Delta}^{2}$ (empty circles) or $\widehat{\Delta}=\widehat{\Delta}$ (filled circles). The arrow indicates the mean quantity $\left\langle\bar{\rho} \sigma_{Z}\right\rangle$.
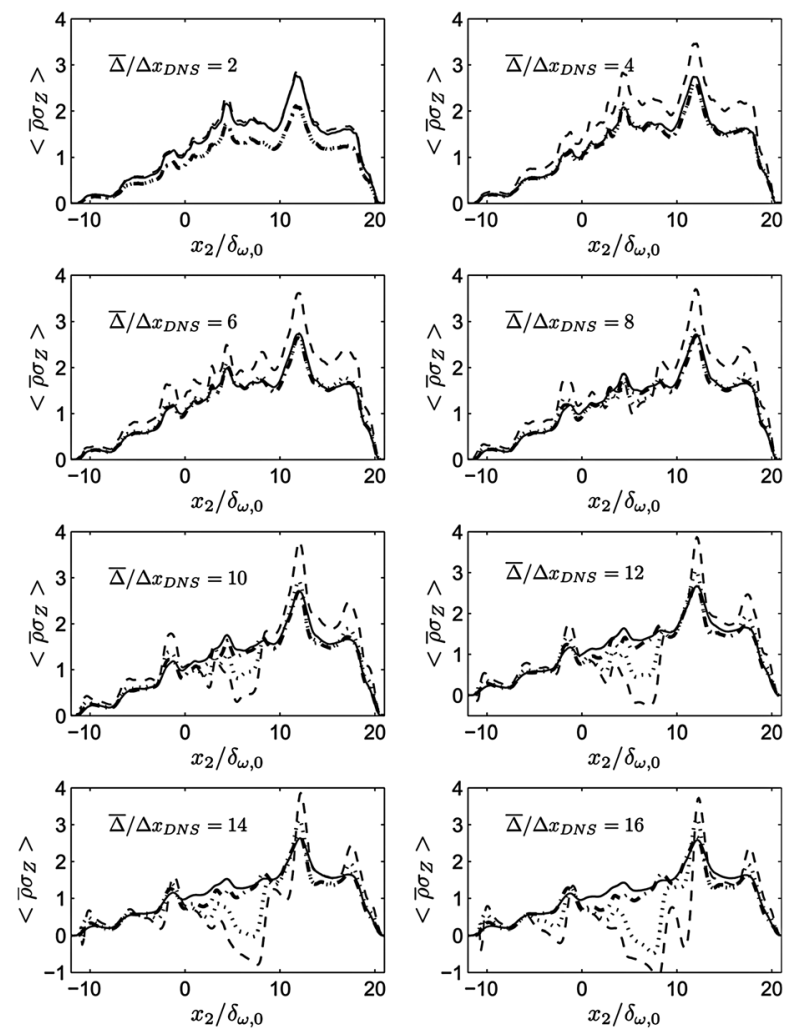

FIG. 24. Profiles of modeled and exact SGS scalar variances evaluated using the filtered OHe600 DNS at $t_{t r}^{*}$. Exact values: solid line. New model: dash-dotted line. Classical model using $\bar{\Delta}^{2}=\widehat{\Delta}^{2}+\bar{\Delta}^{2}$ : dotted line. Classical model using $\bar{\Delta}=\widehat{\Delta}$ : dashed line. Variances are non-dimensionalized by the exact value at the center of the mixing layer.
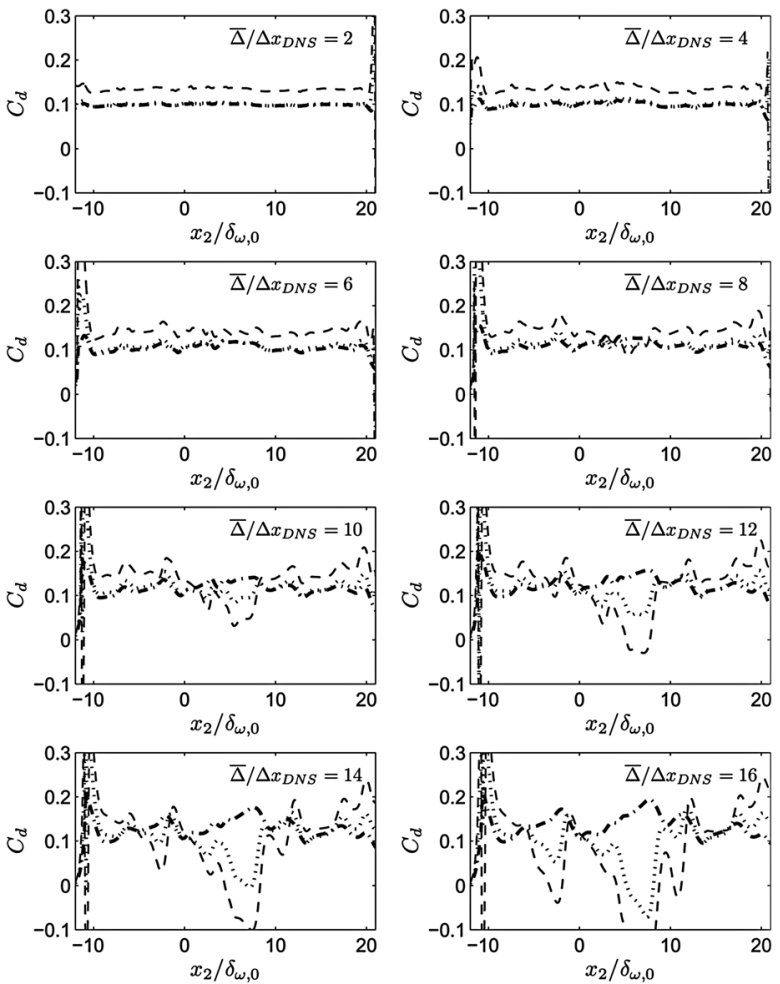

FIG. 25. Model coefficients $C_{d}$ evaluated by using the filtered OHe600 DNS at $t_{t r}^{*}$. New model: dash-dotted line. Classical model using $\widehat{\Delta}^{2}=\widehat{\Delta}^{2}+\bar{\Delta}^{2}$ : dotted line. Classical model using $\widehat{\bar{\Delta}}=\widehat{\Delta}$ : dashed line. 

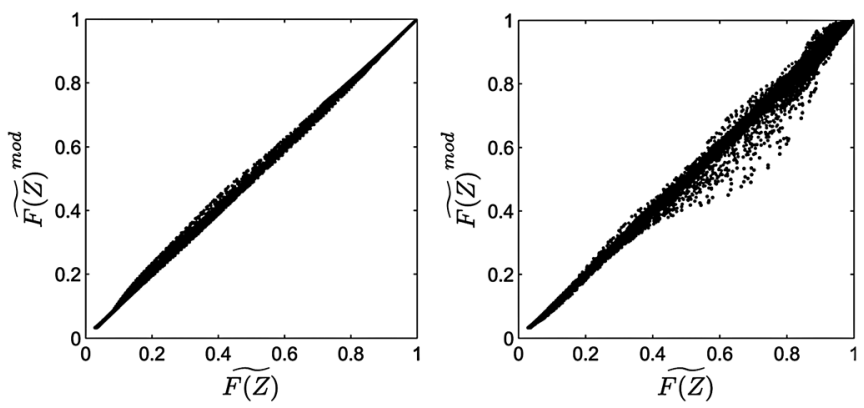

FIG. 26. Scatter plot of the modeled function $\widetilde{F(Z)}$ mod versus the exact quantity $F(Z)$ computed over the plane $x_{2} / \delta_{\omega, 0}=0.44$. The evaluation is made for the filtered HN600 DNS at $t_{t r}^{*}$, using the scalar variance $\sigma_{Z}$ modeled employing a third-order ADM (left), and the new dynamic model (right). $\bar{\Delta} / \Delta x_{D N S}=8$.

Corresponding results for the $\mathrm{OH} 750$ database are displayed in Figs. 20, 21, and 22, and for the OHe600 database are depicted in Figs. 23, 24, and 25. For the OH750 conditional averages and mean profiles, the advantage of the new model predictions are less drastic when compared to the classical model than for the HN600 database. The new model and the classical one with the correct filter width are in close agreement, while their results differ from those using the classical procedure with the incorrect filter width $\bar{\Delta}=\widehat{\Delta}$. For the OHe600 database which has the highest $\operatorname{Re}_{t r}$ value among the three examined, the results at small to moderate $\bar{\Delta} / \Delta x_{D N S}$ values are the same as for HN600 for the $x_{2} / \delta_{\omega, 0}=0.44$ conditional averages; however, for large
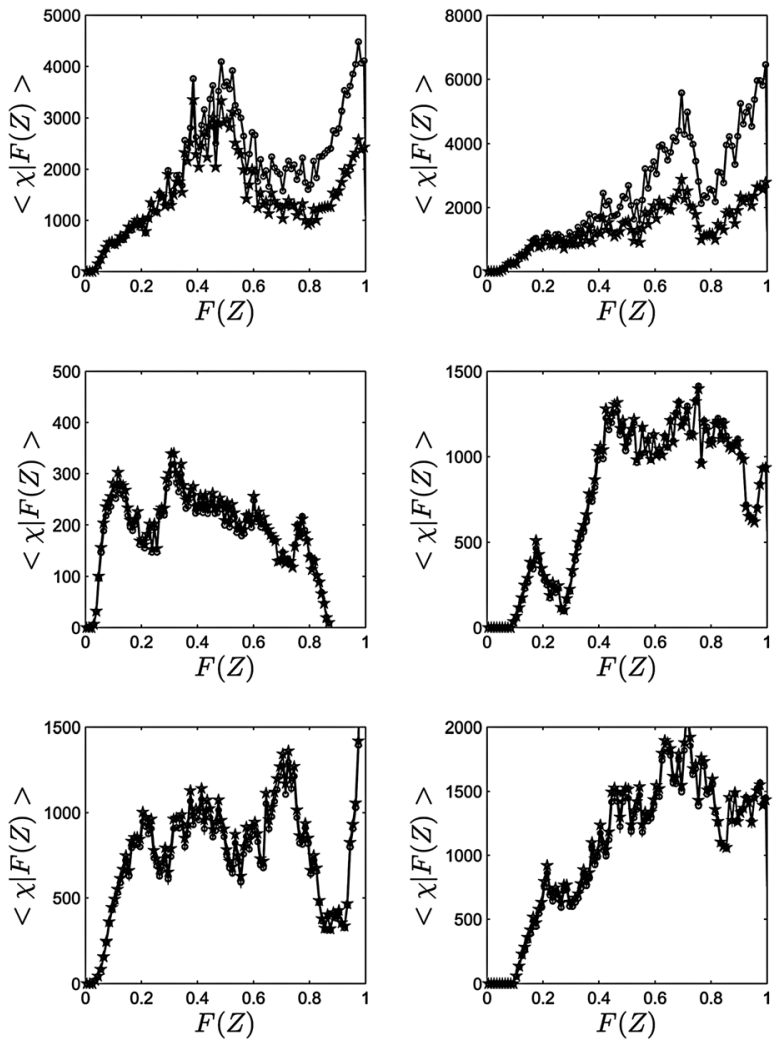

FIG. 27. Conditional average of $\chi_{T}$ (circles) and $\chi_{F}$ (stars) on $F(Z)$ given by Eq. (51). The computations used the HN600 (first row), OH750 (second row), and $\mathrm{OHe} 600$ (third row) DNS at $t_{t r}^{*}$ over planes corresponding to $x_{2} / \delta_{\omega, 0}=0.44$ (left) and $x_{2} / \delta_{\omega, 0}=5.11$ (right).
$\bar{\Delta} / \Delta x_{D N S}$ values close to the production range the predictions from all three models at this particular $x_{2}$-coordinate converge and underestimate the exact value. The advantage of the new model is though evident for predicting the mean SGS variance value (Fig. 24) over all $x_{2}$-planes of the mixing layer even at large $\bar{\Delta} / \Delta x_{D N S}$ values.

\section{Modeled scalar variance in conjunction with the presumed-PDF approach}

As a recall, the results of Fig. 8 were obtained with the exact moments of the SGS PDF, as extracted from the filtered DNS database. It is thus important to explore the potential of the two SGS-scalar-variance models examined in Sec. VI C, namely the ADM using a third-order approximation and the new dynamic gradient-like model. To this end, we use each of these models to construct the $\beta$-PDF and assess their performance in reproducing the same filtered non-linear $Z$-function of Eq. (51), as in Sec. VI B. Predictions are illustrated in Fig. 26 for HN600 at $\bar{\Delta} / \Delta x_{D N S}=8$ and $x_{2} / \delta_{\omega, 0}=0.44$, as an example. Each of the models is evaluated through scatter plots. Independent of the SGS-scalarvariance model used, the predictions are excellent, showing a priori the potential of the new $\sigma_{Z}$ models combined with a PDF approach to reproduce filtered non-linear functions of the scalar, as would be models of the dissipation rate, reaction rates, etc. (non-linear functions that include strong derivatives with respect to the scalar were not tested). Examining the scatter plots, the ADM results show a smaller dispersion than the gradient-based model. On the other hand, the former is more expensive than the latter and the ADM exhibits convergence problems of the deconvolution series for compressible flow, casting uncertainty on success at high Reynolds numbers where convergence will be further influenced by turbulence-dependent aspects.

\section{SUMMARY AND CONCLUSIONS}

The goal of this study was to investigate the modeling of the SGS scalar variance under supercritical-pressure conditions where the real-gas equation of state, the full (3-term) expression for the species mass diffusion flux and transport properties varying with the thermodynamic variables, all preclude assuming that the same models as those at atmospheric-pressure conditions are valid. To this end, we followed the classical approach whereby the SGS scalar variance equation is derived and terms non-computable from the LES solution are modeled, with the intent of providing closure and solving the equation. Thus, we first developed the equation describing the evolution of the SGS scalar variance under supercritical-pressure conditions and highlighted its complexity, particularly recognizing terms not present under atmospheric-pressure conditions. We also presented a second form of the equation which was more adept at highlighting the nature of the new SGS terms and their contributions. The activity of terms in the first form of the equation was examined using a filtered DNS database represented by transitional states describing the mixing of binary species, for three systems of species, under supercritical pressure conditions. The findings were that the activity of some of 
these new terms is of same magnitude as that of classical terms, meaning that they cannot be neglected. Most important among these new terms were those expressing subgrid activity due to spatially variable diffusion coefficients. The second form of the equation confirmed the importance of the SGS diffusivity and identified for this equation a new dissipation contribution arising from the Soret term. Recognizing that no SGS models are available to model these terms, and thus to close the SGS scalar variance equation, the attention was instead refocused on a second method, that is, the direct modeling of the SGS scalar variance.

This second route first involved examining the SGS PDF of the scalar by assuming the form of the PDF and using the same filtered DNS database to extract the exact moments of the PDF. Three PDF forms were investigated - the Dirac, Gaussian, and $\beta$ PDF - and the results showed that they ranked in increasing success in the order cited. This encouraging ability of the $\beta$ PDF motivated the development of two direct models for the SGS scalar variance. The first SGS-scalar-variance model was based on the ADM procedure reformulated for application to compressible flows. The second SGS-scalar-variance model was based on a gradient-like dynamic model using the Leonard term expansion. Success with these two models motivated a reassessment of the ability to model a filtered non-linear function of the scalar by using the $\beta \mathrm{PDF}$ in conjunction with either one of these models for the SGS scalar variance and with the mean computed from the filtered DNS. The findings were that either one of the direct SGS scalar variance models provided a high-fidelity duplication of the DNS-extracted SGS PDF, which is manifested by the excellent reproduction of a filtered non-linear function of the scalar. Although the ADM was generally more accurate than the gradient-based model, it was shown that the ADM procedure is not necessarily convergent for compressible flows; thus, the results could be interpreted as an approximation rather than a model the accuracy of which asymptotically increases with series higher truncation order.

Therefore, although supercritical-pressure conditions entail new challenges in the modeling of the SGS scalar variance, these challenges were met for describing the mixing of several binary-species systems. Further a posteriori studies should reveal the true potential of these models, and applications to reacting flows would represent the ultimate test.

\section{ACKNOWLEDGMENTS}

This study was conducted at the Jet Propulsion Laboratory (JPL) of the California Institute of Technology (Caltech) under sponsorship of the U.S. Department of Energy and of the U.S. Air Force Office of Scientific Research. Computational resources were provided by the supercomputing facility at JPL.

\section{APPENDIX: ASSESSMENT OF THE ONE- DIMENSIONAL LAMINAR SCALAR DISSIPATION FOR TRANSITIONAL SUPERCRITICAL-PRESSURE MIXING LAYERS}

The dissipation rate in the context of the flamelet model is often modeled in the framework of a one-dimensional counterflow. ${ }^{48}$ Under this assumption, an analytical solution for the dissipation rate is available under subcritical conditions ${ }^{1}$ which is the functional form of Eq. (51). In order to evaluate whether this form still holds in the present case, the averages of both $\chi_{T}$ and $\chi_{F}$ of Eqs. (49) and (50) are computed from the DNS conditioned on $F(Z)$ (Eq. (51)). The results are illustrated in Fig. 27. For both $\chi_{T}$ and $\chi_{F}$, there is a linear dependency on $F(Z)$ in zones corresponding to small values of $F(Z)$ but not elsewhere (e.g., for HN600 the linear relationship approximately holds from the smallest $F(Z)$ location where the conditional average is non-null up to approximately 0.38 for $x_{2} / \delta_{\omega, 0}=0.44$ and 0.2 for $\left.x_{2} / \delta_{\omega, 0}=5.11\right)$. As a preamble to interpreting Fig. 27, we note that $F(Z)$ has a bell shape with the maximum corresponding to $Z=0.5$ which indicates perfect mixing, and the minima at either $Z=0$ or $Z=1$ both of which correspond to the pure species. Considering Figs. 4, 5, and 6, it is clear that small values of $F(Z)$ correspond to large $Z$. Thus, for both $\chi_{T}$ and $\chi_{F}$, there is a linear dependency on $F(Z)$ in zones corresponding to either small or large values of $Z$, while departures are observed for intermediary values of $Z$. This indicates that $F(Z)$ is unreliable since it cannot handle regions of fluid mixing where the gradients would be largest.

${ }^{1}$ N. Peters, Turbulent Combustion (Cambridge University Press, Cambridge, UK, 2000).

${ }^{2}$ A. W. Cook and J. Riley, "A subgrid model for equilibrium chemistry in turbulent flows," Phys. Fluids 6, 2868 (1994).

${ }^{3}$ J. Jiménez, A. Linan, M. M. Rogers and F. J. Higuera, "A priori testing of subgrid models for chemically reacting non-premixed turbulent shear flows," J. Fluid Mech. 349, 149 (1997).

${ }^{4}$ C. D. Pierce and P. Moin, "A dynamic model for subgrid variance and dissipation rate of a conserved scalar," Phys. Fluids 10(12), 3041 (1998).

${ }^{5}$ G. Balarac, H. Pitsch, and V. Raman, "Development of a dynamic model for the subfilter scalar variance using the concept of optimal estimators," Phys. Fluids 20, 035114 (2008).

${ }^{6}$ C. Jiménez, F. Ducros, B. Cuenot, and B. Bédat, "Subgrid scale variance and dissipation of a scalar field in large eddy simulations," Phys. Fluids 13(6), 1748 (2001).

${ }^{7}$ C. Pera, J. Réveillon, L. Vervisch, and P. Domingo, "Modeling subgrid scale mixture fraction variance in LES of evaporating spray," Combust. Flame 146, 635 (2006).

${ }^{8}$ N. A. Okong'o and J. Bellan, "Direct numerical simulation of a transitional supercritical binary mixing layer: Heptane and nitrogen," J. Fluid Mech. 464, 1 (2002).

${ }^{9}$ L. C. Selle, N. A. Okong'o, J. Bellan, and K. G. Harstad, "Modelling of subgrid-scale phenomena in supercritical transitional mixing layers: An a priori study," J. Fluid Mech. 593, 57 (2007).

${ }^{10} \mathrm{~N}$. Okong'o, K. Harstad, and J. Bellan, "Direct numerical simulations of $\mathrm{O}_{2} / \mathrm{H}_{2}$ temporal mixing layers under supercritical conditions," AIAA J. 40(5), 914 (2002).

${ }^{11}$ N. A. Okong'o and J. Bellan, "Turbulence and fluid-front area production in binary-species, supercritical, transitional mixing layers," Phys. Fluids 16(5), 1467 (2004).

${ }^{12}$ E. S. Taşkinoğlu and J. Bellan, "A posteriori study using a DNS database describing fluid disintegration and binary-species mixing under supercritical pressure: Heptane and nitrogen," J. Fluid Mech. 645, 211 (2010).

${ }^{13}$ N. A. Okong'o and J. Bellan, "Entropy production of emerging turbulent scales in a temporal supercritical n-heptane/nitrogen three-dimensional mixing layer," Proc. Combust. Inst. 28, 497 (2000).

${ }^{14} \mathrm{H}$. Pitsch and H. Steiner, "Large-eddy simulation of a turbulent piloted methane-air diffusion flame (Sandia flame D)," Phys. Fluids 12(10), 2541 (2000).

${ }^{15}$ V. John, I. Angelov, A. A. Öncül, and D. Thévenin, "Techniques for the reconstruction of a distribution from a finite number of its moments," Chem. Eng. Sci. 62, 2890 (2007).

${ }^{16} \mathrm{C}$. Pantano and S. Sarkar, "A subgrid model for nonlinear functions of a scalar,” Phys. Fluids 13, 3803 (2001). 
${ }^{17}$ J. P. Mellado, S. Sarkar, and C. Pantano, "Reconstruction subgrid models for nonpremixed combustion," Phys. Fluids 15, 3280 (2003).

${ }^{18}$ S. B. Pope, "PDF methods for turbulent reactive flows," Prog. Energy Combust. Sci. 11, 119 (1985).

${ }^{19}$ P. J. Colucci, F. A. Jaberi, and P. Givi, "Filtered density function for large eddy simulation of turbulent reacting flows," Phys. Fluids 10(2), 499 (1998).

${ }^{20} \mathrm{H}$. Steiner and W. K. Bushe, "Large eddy simulation of a turbulent reacting jet with conditional source-term estimation,” Phys. Fluids 13(3), 754 (2001).

${ }^{21}$ F. A. Jaberi, P. J. Colucci, S. James, P. Givi, and S. B. Pope, "Filtered mass density function for large-eddy simulation of turbulent reacting flows," J. Fluid Mech. 401, 85 (1999).

${ }^{22}$ V. Raman, H. Pitsch, and R. O. Fox, "Hybrid large-eddy simulation/Lagrangian filtered-density-function approach for simulating turbulent combustion," Combust. Flame 143, 56 (2005).

${ }^{23}$ R. Fox, Computational Models for Turbulent Reacting Flows (Cambridge University Press, Cambridge, 2003).

${ }^{24} \mathrm{H}$. Pitsch, "Large-eddy simulation of turbulent combustion," Annu. Rev. Fluid Mech. 38, 453 (2006)

${ }^{25}$ A. Moreau, O. Teytaud, and J. P. Bertoglio, "Optimal estimation for largeeddy simulation of turbulence and application to the analysis of subgrid models," Phys. Fluids 18, 105101 (2006).

${ }^{26}$ E. A. Brizuela and R. W. Bilger, "On the eddy break-up coefficient," Combust. Flame 104, 208 (1996).

${ }^{27} \mathrm{P}$. Sagaut, Large-Eddy Simulation for Incompressible Flows: An Introduction (Springer-Verlag, Berlin/New York, 2001).

${ }^{28}$ S. Stolz and N. A. Adams, "An approximate deconvolution procedure for large-eddy simulation," Phys. Fluids 11, 1699 (1999).

${ }^{29}$ P. H. van Cittert, "Zum Einfluss der Spaltbreite auf die Intensitatswerteilung in Spektrallinien ii," Zeitschrift für Physik 69, 298 (1931).

${ }^{30}$ J. A. Domaradzki and N. A. Adams, "Direct modelling of subgrid scales of turbulence in large eddy simulations," J. Turbul. 3(907746985), N24 (2002).

${ }^{31}$ S. Stolz, N. A. Adams, and L. Kleiser, "An approximate deconvolution model for large-eddy simulation with application to incompressible wallbounded flows," Phys. Fluids 13(4), 997 (2001).

${ }^{32} \mathrm{~S}$. Stolz, N. A. Adams, and L. Kleiser, "The approximate deconvolution model for large-eddy simulations of compressible flows and its application to shock-turbulent-boundary-layer interaction," Phys. Fluids 13(10), 2985 (2001).
${ }^{33}$ T. Dubois, J. A. Domaradzki, and A. Honien, "The subgrid-scale estimation model applied to large eddy simulations of compressible turbulence," Phys. Fluids 14(5), 1781 (2002).

${ }^{34}$ M. Germano, U. Piomelli, P. Moin, and W. H. Cabot, "A dynamic subgrid-scale eddy viscosity model," Phys. Fluids A 3(7), 1760 (1991).

${ }^{35} \mathrm{P}$. Moin, K. Squires, W. H. Cabot, and S. Lee, "A dynamic subgrid-scale model for compressible turbulence and scalar transport," Phys. Fluids A 3, 2746 (1991).

${ }^{36} \mathrm{C}$. Wall, B. J. Boersma, and P. Moin, “An evaluation of the assumed beta probability density function subgrid-scale model for large eddy simulation of nonpremixed, turbulent combustion with heat release," Phys. Fluids 12, 2522 (2000).

${ }^{37}$ S. H. Kim and H. Pitsch, "Mixing characteristics and structure of a turbulent jet diffusion flame stabilized on a bluff-body," Phys. Fluids 18, 075103 (2006).

${ }^{38}$ J. Bardina, J. H. Ferziger, and W. C. Reynolds, "Improved subgrid scale models for large eddy simulation," AIAA Paper 80, 1357 (1980).

${ }^{39}$ J. Réveillon and L. Vervisch, "Response of the dynamic LES model to heat release induced effects," Phys. Fluids 8(8), 2248 (1996).

${ }^{40} \mathrm{P}$. DesJardin and S. Frankel, "Large eddy simulation of a nonpremixed reacting jet: Application and assessment of subgrid-scale combustion models," Phys. Fluids 10(9), 2298 (1998).

${ }^{41} \mathrm{~K}$. Lilly, "A proposed modification of the Germano subgrid-scale closure method," Phys. Fluids A 4, 633 (1992).

${ }^{42}$ M. Germano, "Turbulence: The filtering approach," J. Fluid Mech. 238, 325 (1992).

${ }^{43}$ A. W. Vreman, "Direct and large-eddy simulation of the compressible turbulent mixing layer," Ph.D dissertation (University of Twente, 1995).

${ }^{44}$ K. W. Bedford and W. K. Yeo, Large Eddy Simulation of Complex Engineering and Geophysical Flows, edited by B. Galperin and S. A. Orszag (Cambridge University Press, New York, 1993).

${ }^{45} \mathrm{C}$. Brun and R. Friedrich, "Modeling the test SGS tensor Tij: An issue in the dynamic approach," Phys. Fluids 13(8), 2373 (2001).

${ }^{46} \mathrm{~B}$. Vreman, B. Geurts, and H. Kuerten, "On the formulation of the dynamic mixed subgrid scale model," Phys. Fluids 6, 4057 (1994).

${ }^{47}$ A. W. Cook and J. Riley, "Subgrid-scale modeling for turbulent reacting flows," Combust. Flame 112, 593 (1998).

${ }^{48}$ A. W. Cook, J. J. Riley, and G. A. Kosály, "Laminar flamelet approach to subgrid-scale chemistry in turbulent flows," Combust. Flame 109, 332 (1997). 